This is an electronic reprint of the original article. This reprint may differ from the original in pagination and typographic detail.

\author{
Author(s): Honkavaara, Eija; Eskelinen, Matti; Pölönen, Ilkka; Saari, Heikki; Ojanen, Harri; \\ Mannila, Rami; Holmlund, Christer; Hakala, Teemu; Litkey, Paula; Rosnell, Tomi; \\ Viljanen, Niko; Pulkkanen, Merja
}

Title: $\quad$ Remote Sensing of 3-D Geometry and Surface Moisture of a Peat Production Area Using Hyperspectral Frame Cameras in Visible to Short-Wave Infrared Spectral Ranges Onboard a Small Unmanned Airborne Vehicle (UAV)

Year: $\quad 2016$

Version:

Please cite the original version:

Honkavaara, E., Eskelinen, M., Pölönen, I., Saari, H., Ojanen, H., Mannila, R., Holmlund, C., Hakala, T., Litkey, P., Rosnell, T., Viljanen, N., \& Pulkkanen, M. (2016). Remote Sensing of 3-D Geometry and Surface Moisture of a Peat Production Area Using Hyperspectral Frame Cameras in Visible to Short-Wave Infrared Spectral Ranges Onboard a Small Unmanned Airborne Vehicle (UAV). IEEE Transactions on Geoscience and Remote Sensing, 54(9). https://doi.org/10.1109/TGRS.2016.2565471

All material supplied via JYX is protected by copyright and other intellectual property rights, and duplication or sale of all or part of any of the repository collections is not permitted, except that material may be duplicated by you for your research use or educational purposes in electronic or print form. You must obtain permission for any other use. Electronic or print copies may not be offered, whether for sale or otherwise to anyone who is not an authorised user. 


\title{
Remote Sensing of 3-D Geometry and Surface Moisture of a Peat Production Area Using Hyperspectral Frame Cameras in Visible to Short-Wave Infrared Spectral Ranges Onboard a Small Unmanned Airborne Vehicle (UAV)
}

\author{
Eija Honkavaara, Matti A. Eskelinen, Ilkka Pölönen, Heikki Saari, Harri Ojanen, Rami Mannila, Christer Holmlund, \\ Teemu Hakala, Paula Litkey, Tomi Rosnell, Niko Viljanen, and Merja Pulkkanen
}

\begin{abstract}
Miniaturized hyperspectral imaging sensors are becoming available to small unmanned airborne vehicle (UAV) platforms. Imaging concepts based on frame format offer an attractive alternative to conventional hyperspectral pushbroom scanners because they enable enhanced processing and interpretation potential by allowing for acquisition of the 3-D geometry of the object and multiple object views together with the hyperspectral reflectance signatures. The objective of this investigation was to study the performance of novel visible and near-infrared (VNIR) and short-wave infrared (SWIR) hyperspectral frame cameras based on a tunable Fabry-Pérot interferometer (FPI) in measuring a 3-D digital surface model and the surface moisture of a peat production area. UAV image blocks were captured with ground sample distances (GSDs) of 15, 9.5, and $2.5 \mathrm{~cm}$ with the SWIR, VNIR, and consumer RGB cameras, respectively. Georeferencing showed consistent behavior, with accuracy levels better than GSD for the FPI cameras. The best accuracy in moisture estimation was obtained when using the reflectance difference of the SWIR band at $1246 \mathrm{~nm}$ and of the VNIR band at $859 \mathrm{~nm}$, which gave a root mean square error (rmse) of $5.21 \mathrm{pp}$ ( $\mathrm{pp}$ is the mass fraction in percentage points) and a normalized rmse of $7.61 \%$. The results are encouraging, indicating that UAV-based remote sensing could significantly improve the efficiency and environmental safety aspects of peat production.
\end{abstract}

Manuscript received October 21, 2015; revised February 2, 2016; accepted March 31, 2016. This work was supported by the Finnish Funding Agency for Technology and Innovation Tekes through the HSI-Stereo project and the Measurement, Monitoring and Environmental Efficiency Assessment research program coordinated by Cleen Ltd.

E. Honkavaara, T. Hakala, P. Litkey, T. Rosnell, and N. Viljanen are with the Department of Remote Sensing and Photogrammetry, Finnish Geospatial Research Institute, 02430 Masala, Finland (e-mail: eija.honkavaara@nls.fi; teemu.hakala@nls.fi; paula.litkey@nls.fi; tomi.rosnell@nls.fi; niko.viljanen@ nls.fi).

M. A. Eskelinen and I. Pölönen are with the Department of Mathematical Information Technology, University of Jyväskylä, 40014 Jyväskylä, Finland (e-mail: matti.a.eskelinen@student.jyu.fi; ilkka.polonen@jyu.fi).

H. Saari, H. Ojanen, R. Mannila, and C. Holmlund are with VTT Microelectronics, 02044 Espoo, Finland (e-mail: heikki.saari@vtt.fi; harri.ojanen@vtt.fi; rami.mannila@vtt.fi; christer.holmlund@vtt.fi).

M. Pulkkanen is with Vapo Oy Clean Waters, 40100 Jyväskylä, Finland (e-mail: merja.pulkkanen@vapo.fi).

Color versions of one or more of the figures in this paper are available online at http://ieeexplore.ieee.org.

Digital Object Identifier 10.1109/TGRS.2016.2565471
Index Terms-Calibration, geographic information system, geometry, image classification, radiometry, remote sensing, remotely piloted aircraft, spectroscopy, stereo vision.

\section{INTRODUCTION}

$\mathbf{R}$ EMOTE sensing using small unmanned airborne vehicles (UAVs) is a rapidly emerging technology. UAV-based remote sensing offers possibilities for cost-efficient data collection with desired spatial and temporal resolutions, which opens up completely new remote sensing applications and new possibilities to perform scientific studies in our environment [1], [2].

An appropriate sensor is a fundamental component of a UAV remote sensing system. The first operational, civil, and lightweight UAV imaging systems typically used commercial video cameras or still cameras operating in three widebandwidth bands in red, green, blue (RGB) and/or near-infrared spectral regions [3]-[5]. Miniaturized hyperspectral sensors have become available to UAV platforms, offering enhanced possibilities for remote sensing applications. Hyperspectral remote sensing employs tens to hundreds of contiguous bands to accurately reconstruct the spectral signature of the target of interest [6]. The first miniaturized sensors operated in the visible to near-infrared spectral (VNIR) range extending to approximately 400-1000 nm. Several pushbroom-type hyperspectral sensors have recently been implemented in UAVs [7]-[11]. Researchers have also implemented point-based spectrometers in UAVs [12], [13]. Lately, novel hyperspectral cameras operating in a frame format principle have entered the market, such as the Rikola Hyperspectral Camera (http://www.rikola.fi), Cubert UHD 185-Firefly (http://cubert-gmbh.de/), or the IMEC SM5X5 (http://www2.imec.be). The frame sensors can be further classified based on the imaging principle as ones capturing all bands simultaneously (snapshot imaging) or as those capturing unregistered bands [14]. The methods for capturing images with unregistered bands include the time-sequential principle or multiple cameras.

When considering different sensing principles, the advantages of the frame imaging approach include the possibility to collect image blocks with stereoscopic multiple object views 


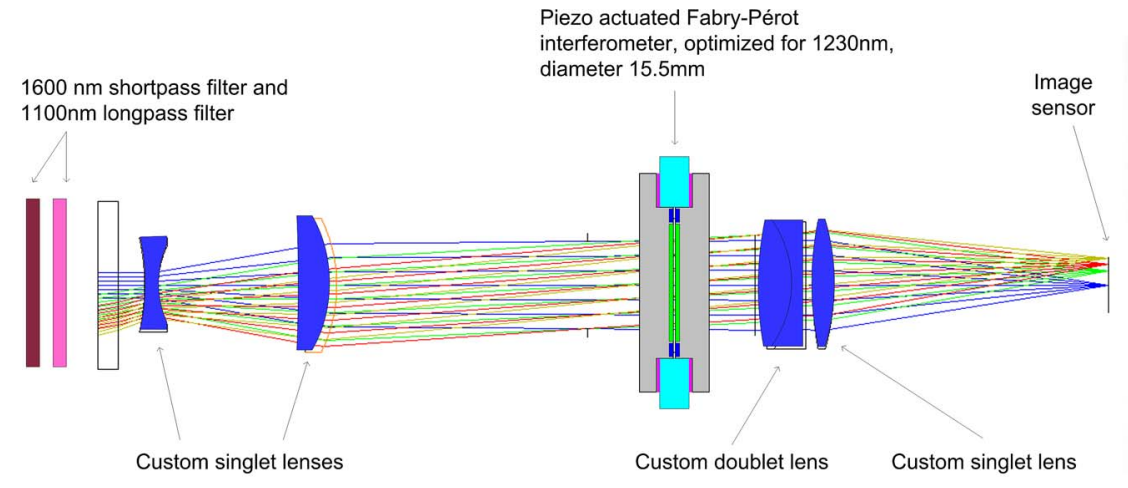

(a)

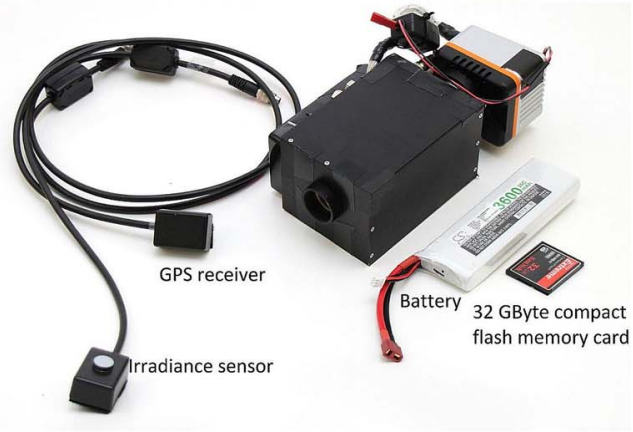

(b)

Fig. 1. (a) Principle of the optical system of FPI SWIR camera prototype 2014. (b) Components of the FPI SWIR imaging system.

and the geometric and radiometric constraints provided by the rigid rectangular image geometry and multiple overlapping images [14], [15]. These can be seen as important advantages in comparison to classical hyperspectral imaging methods based on the pushbroom scanning technology or on point-based spectral measurements. This is valuable in particular for UAV applications, which typically operate under dynamic, vibrating, and turbulent conditions. Furthermore, in many applications, 3-D information is a significant feature parallel to spectral information.

This study investigates novel hyperspectral imaging technology based on a variable air gap Fabry-Pérot interferometer (FPI). The FPI technology makes it possible to manufacture a lightweight frame format hyperspectral imager operating on the time-sequential principle. The first prototypes of the FPIbased cameras were operating in the VNIR spectral range [16]-[18], and recently, a short-wave infrared (SWIR) region prototype operating in the spectral range of $1100-1600 \mathrm{~nm}$ was presented [19]. The FPI technology has also become commercially available in the VNIR range (http://www.rikola.fi). In the UAV operation, the camera is operated using photogrammetric principles, capturing image blocks with stereoscopic overlaps. It is crucial to emphasize the data postprocessing steps that are required to transform these huge amounts of images into products that allow the objects' geometric and spectral characteristics to be interpreted on a quantitative geometric and radiometric basis. Recently, the usability of stereoscopic frame images has improved to a new level due to the development of the structure-from-motion-based image orientation techniques [20] and the dense digital matching technologies generating accurate 3-D point clouds and digital surface models (DSMs) [21]-[26]. These modern computer vision and photogrammetric techniques are capable of providing high-quality 3-D geometric data in a highly automated way.

Surface moisture is one of the key parameters in various environmental and hydrological applications, such as agricultural water management and catchment management [27]-[29]. This work investigates the potential of FPI sensors in estimating surface moisture of a peat production area aiming at improving the efficiency and safety aspects of peat production. When harvesting peat for energy production, peat surface moisture is a critical parameter [30]. Peat is used in energy production mainly in countries where large mires can be found, such as
Finland, Sweden, Russia, and Ireland. Before using peat in a burner, it is first ground from the mire surface. When the peat grind is dry enough, it is harvested in large stacks to wait for transportation to the heating plant. Peat should be suitably dry to improve combustion at the heating plant, while avoiding spontaneous combustion in the stack. Currently, the moisture measurements are carried out by collecting samples of peat and measuring the wet and dry weight of the samples, lasting for at least $24 \mathrm{~h}$. With the UAV remote-sensing-based method, the speed and efficiency of peat moisture measurement could be remarkably improved. Previous investigation showed that peat spectra had several features particularly in the SWIR region supporting classification of moisture and humification levels of peat [31]. Thermal imaging is also a potential technology for surface moisture estimation. Laboratory measurements have indicated good potential of this technology, but previous experimental results with a thermal camera from a manned aircraft platform showed weak correlation of surface moisture and temperature [30].

The objective of this investigation was to study the performance of novel FPI-based VNIR and SWIR hyperspectral frame cameras in generating a DSM and measuring surface moisture of a peat production area. The expectation was that the SWIR range data are more suitable for moisture estimation than the VNIR data, but it was of interest to compare both spectral ranges. We depict the FPI camera technology in Section II. We describe the test setup used for the empirical investigation in Section III, present the empirical results in Section IV, and discuss them in more detail in Section V.

\section{FPI Spectral CAMERA Technology}

\section{A. Principle of FPI-Based Spectral Imager}

The hyperspectral camera developed at the VTT Technical Research Centre of Finland (VTT) [16]-[19] is based on the use of multiple orders of a variable air-gap FPI. When the FPI is placed in front of the sensor, the wavelength of the light passing the FPI is a function of the interferometer air gap (see Fig. 1). By changing the air gap, it is possible to acquire a new set of wavelengths. The final spectral response is dependent on the light passing the FPI and the spectral characteristics of the detector. The spectral bands can be selected according to the requirements of the remote sensing task. In various 
implementations, three-color [15]-[18] or single-color sensors have been used (http://www.rikola.fi). The number of transmission peaks passing the FPI is one to three; thus, exposure with a single gap width provides one to three different spectral bands, when a three-color sensor is used [16]-[18]. With a single-color sensor, one spectral band is obtained for each air-gap value. The first FPI cameras operated in the VNIR range, and recently, Mannila et al. [19] presented the first implementation of the FPI technology in the SWIR range. The first photogrammetric and remote sensing data analyses with the FPI hyperspectral imaging technology have shown that it has excellent potential in remote sensing [15], [32]-[35].

During data collection, a predefined sequence of air-gap values is applied to capture the full spectral range. The hyperspectral data cube is thus formed in the time-sequential imaging principle. When using this technology on a moving platform, each band in the data cube exposed to a different air-gap value has a slightly different position and orientation, which has to be taken into account in the postprocessing phase. During the flight, the integration time, gain, and FPI air-gap information are stored. FPI cameras are equipped with a Global Positioning System (GPS) receiver that records the exact time of the beginning of each data cube; furthermore, the sensor electronics output the synchronization pulse of each exposure (which have not been utilized thus far). An irradiance sensor based on the Intersil ISL29004 photodetector with a spectral sensitivity range of $400-1000 \mathrm{~nm}$ is integrated in the camera to measure the irradiance during each exposure. The sensor is not calibrated; thus, relative broadband irradiance intensity values are obtained [15], [36]. The dark signal is collected before the flight. All data are applied to the images after the flight in the postprocessing phase, as described in Section III-C-E.

\section{B. Cameras Used in This Investigation}

The FPI camera prototype 2012b belonging to the Finnish Geospatial Research Institute was used to capture VNIR images [15], [18]. It is equipped with custom optics having a focal length of $10.9 \mathrm{~mm}$ and an f-number of 2.8. The camera has a CMOSIS CMV4000 RGB image sensor with an electronic shutter. The time difference between adjacent exposures is $0.075 \mathrm{~s}$, giving a time difference between the first and last exposures in a data cube with 24 bands of $1.8 \mathrm{~s}$. The sensor is used in a twice binned mode, providing an image size of $1024 \times 648$ pixels with a pixel size of $11 \mu \mathrm{m}$. The field of view (FOV) is $\pm 18^{\circ}$ in the flight direction, $\pm 27^{\circ}$ in the cross-flight direction, and $\pm 31^{\circ}$ at the format corner. The entire camera system weighs less than $700 \mathrm{~g}$.

The SWIR range spectral imager consists of the commercial indium gallium arsenide (InGaAs) camera-the Xenics Bobcat-1.7-320, the imaging optics, the FPI module, control electronics, a battery, a GPS sensor, and an irradiance sensor (see Fig. 1) [19]. The Xenics Bobcat-1.7-320 is an uncooled InGaAs camera, with a spectral band of $0.9-1.7 \mu \mathrm{m}$ and $320 \times$ 256 pixels and a pixel size of $20 \times 20 \mu \mathrm{m}$. The FPI, optics, and electronics are designed and built at VTT. The focal length of the optics is $12.2 \mathrm{~mm}$, and the f-number is 3.2 ; the FOV is $\pm 13^{\circ}$ in the flight direction, $\pm 15.5^{\circ}$ in the cross-flight direction,

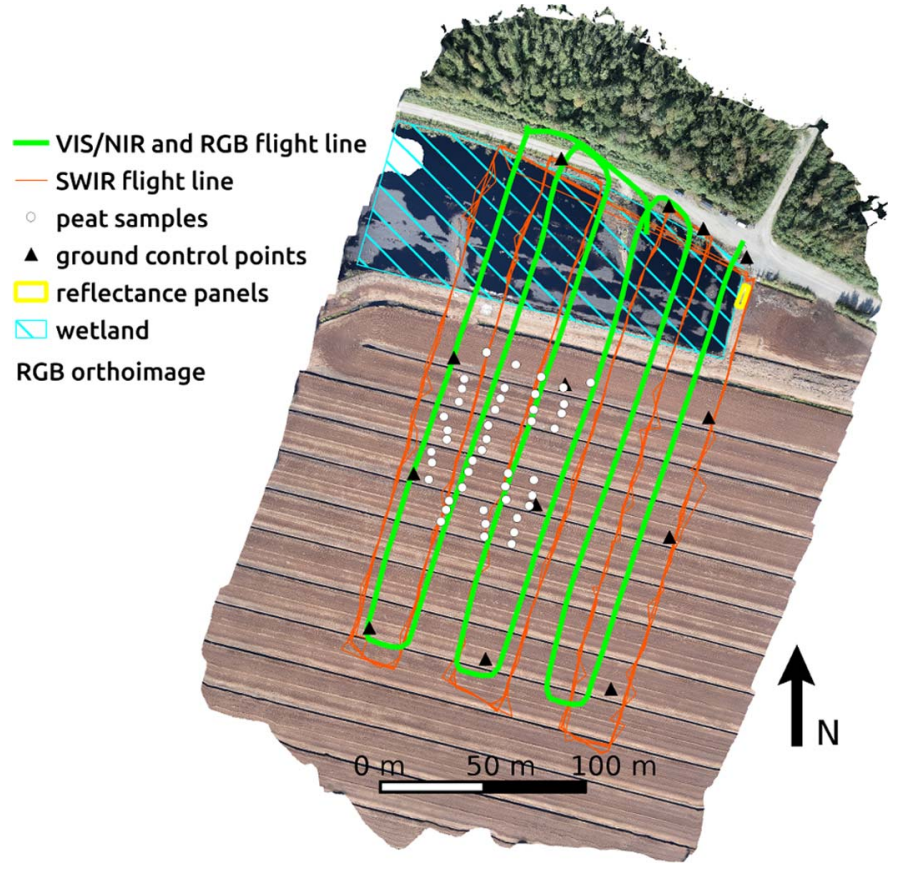

Fig. 2. Flight lines, ground control points (GCPs), and distribution of peat samples.

and $\pm 20^{\circ}$ at the format corner. The time between adjacent exposures is $10 \mathrm{~ms}$ plus exposure time; capturing single data cube with 32 bands and using 2-ms exposure time takes $0.384 \mathrm{~s}$. The mass of the spectral imager unit is approximately $1200 \mathrm{~g}$.

\section{MAterials AND Methods}

\section{A. Test Area and Flight Campaign}

Test flights were carried out in Okssuo in southern Finland $\left(60^{\circ} 49^{\prime} 24.534^{\prime \prime}, 23^{\circ} 56^{\prime} 12.325^{\prime \prime}\right)$ on 11 September 2014. The study site is the peat production area of Vapo Oy, having a flat topography with parallel ditches and covered by spectrally homogeneous peat (see Fig. 2). The size of the area of interest was about $150 \mathrm{~m} \times 150 \mathrm{~m}$. Weather conditions were sunny, clear, and windless.

Image blocks with six image strips (see Fig. 2) were collected using three different cameras: the FPI VNIR camera prototype 2012b, the new FPI SWIR camera prototype, and a commercial RGB camera, the Samsung NX300. Samsung NX300 has a $23.5 \times 15.7 \mathrm{~mm}$ CMOS RGB sensor with 20.3 megapixels and a 16-mm lens; it was used to collect high-spatial-resolution stereoscopic data for comparison and reference purposes.

For the FPI SWIR camera, we used an 8-rotor UAV, based on the MikroKopter autopilot and the Droidworx AD-8 extended frame with a $1.5-\mathrm{kg}$ payload capacity. The camera was rigidly mounted to the landing gear of the UAV [see Fig. 3(a)]. The Samsung NX300 and FPI VNIR cameras were simultaneously operated using a hexacopter with a Tarot 960 foldable frame with Tarot 5008 (340 KV) brushless electric motors having a 4-kg payload capacity. The autopilot was Pixhawk equipped with Arducopter 3.15 firmware. Both cameras were rigidly mounted to the payload rails of the hexacopter [see Fig. 3(b)]. In practical operation, it is recommended to install the cameras on stabilized mount to compensate for impacts of the platform 


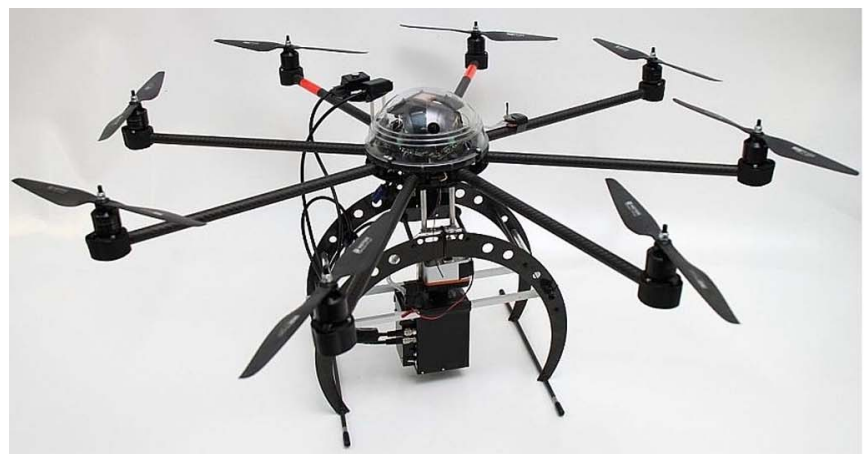

(a)

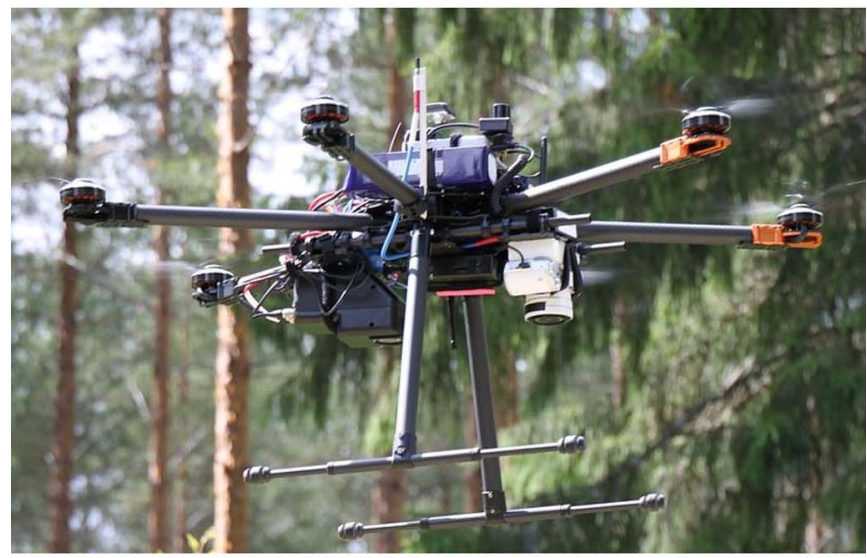

(b)

Fig. 3. (a) Lightweight SWIR camera installed in an octocopter UAV. (b) VNIR and Samsung NX300 cameras installed in a hexacopter UAV.

TABLE I

Details of The IMAGe Blocks. F: FLIGHT DiRection; CF: CROSS-Flight DiRECTION; FOV: FIELD OF VIEW

\begin{tabular}{llll}
\hline \hline Sensor & SWIR & VNIR & RGB \\
\hline Spectral sensitivity (nm) & $1100-1600$ & $500-900$ & R, G, B \\
Flight height (m) & 89 & 94 & 94 \\
GSD (m) & 0.15 & 0.095 & 0.025 \\
Exposure time (ms) & 2 & 15 & 0.5 \\
Footprint f; cf (m) & $38 ; 47$ & $67 ; 106$ & $90 ; 135$ \\
Overlap f, cf (\%) & $77 ; 43$ & $76 ; 78$ & $92 ; 83$ \\
FOV f, cf $\left(^{\circ}\right)$ & $\pm 13^{\circ} ; \pm 15.5^{\circ}$ & $\pm 20 ; \pm 29$ & $\pm 26 ; \pm 36$ \\
Flight Speed (m/s) & 4 & 4 & 4 \\
Number of images & 157 & 117 & 235 \\
Time (UTC +0) & $11: 48-12: 00$ & $11: 19-11: 28$ & $11: 19-11: 28$ \\
Sun elevation; azimuth $\left({ }^{\circ}\right)$ & $31 ; 208$ & $32 ; 199$ & $32 ; 199$ \\
\hline \hline
\end{tabular}

vibrations and fast movements in the image quality. The use of stabilized mount was not possible in this investigation due to experimental setups.

The photogrammetric block setup was designed for the SWIR camera at a flying height of $90 \mathrm{~m}$ above ground level. The resulting ground sample distance (GSD) was $15 \mathrm{~cm}$ for the SWIR camera, $9.5 \mathrm{~cm}$ for the VNIR camera, and $2.5 \mathrm{~cm}$ for the RGB camera. In the case of the SWIR camera, the size of the image footprint was $38 \mathrm{~m} \times 47 \mathrm{~m}$, the forward overlap was $77 \%$, and the side overlap was $43 \%$ on average. For the FPI VNIR camera and the RGB camera, the overlaps were larger (see Table I).

The spectral settings of the FPI VNIR and SWIR cameras were selected so that the spectral range was covered quite evenly (see Table II). A total of 32 spectral bands were collected by the FPI SWIR camera in the spectral range 1100-1600 nm with the full width of half maximum (FWHM) ranging from 20 to $30 \mathrm{~nm}$ and with an exposure time of $2 \mathrm{~ms}$. With the VNIR camera, 38 bands were collected on a spectral range of 500-900 nm having an FWHM of 11-31 nm and an exposure time of $15 \mathrm{~ms}$. The long exposure time was used to obtain a good dynamic range in the relatively dark peat surface (reflectance $<0.3$ ). The bright reflectance panels with the nominal reflectance of 0.5 were saturated with this setting; hence, it is not suitable for applications having reflectance values brighter than 0.5 , for example, vegetation remote sensing. The long exposure time could also cause image quality reduction due to motion blur. However, the movement of the platform was less than the GSD during the exposure, and the weather conditions were excellent, i.e., low winds and no turbulence; thus, no significant motion blur was expected. Visual inspection of images did not show noticeable motion blur.

\section{B. Ground Reference}

We deployed 13 GCPs targeted with circular targets with a diameter of $30 \mathrm{~cm}$ (see Fig. 2). Their coordinates were measured using the virtual reference station real-time kinematic GPS (VRS-GPS) method with accuracy levels [root mean square error (rmse)] of approximately $3 \mathrm{~cm}$ in $\mathrm{X}$ and $\mathrm{Y}$ and $4 \mathrm{~cm}$ in $\mathrm{Z}$ coordinates [37].

For reflectance transformation purposes, reflectance panels of size $1 \mathrm{~m} \times 1 \mathrm{~m}$ and with nominal reflectivity of $0.03,0.1$, and 0.5 were installed in the area. Materials of the panels were carefully selected to provide uniform reflectance properties and low anisotropy; black and dark gray panels were made of carpet, whereas the brightest panel was a painted panel [38]. The reference reflectance values were measured in a laboratory with an estimated accuracy level of $2 \%-5 \%$ using the FIGIFIGO goniospectrometer [39].

Altogether, 44 peat samples of size $0.05 \mathrm{~m} \times 0.05 \mathrm{~m} \times$ $0.03 \mathrm{~m}$ were taken and measured for reflectance and moisture in the laboratory. Spatial locations of samples (XYZ coordinates) were measured with VRS-GPS. Moisture content (MC [\%]) of the samples was determined based on wet weight $\left(w_{\text {wet }}\right)$ and dry weight $\left(w_{\text {dry }}\right)$, i.e.,

$$
M C=100 w_{\text {wet }} /\left(w_{\text {dry }}+w_{\text {wet }}\right)[\%] .
$$

Sample moisture varied from $50.0 \%$ to $78.4 \%$, and the average moisture was $67.4 \%$.

\section{Data Processing}

Hundreds of small-format UAV images were collected to cover the area of interest. Rigorous processing was required to derive quantitative information from the imagery. The processing of FPI camera images is similar to any frame format camera images; the major difference is the processing of the nonoverlapping spectral bands. The FPI data processing line for MC estimation contained the following steps:

1) applying laboratory calibration corrections to the images;

2) determination of the geometric imaging model, including interior and exterior orientations of the images; 
TABLE II

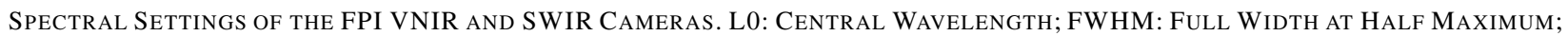
DT: Time Difference to the Start of the Data Cube; DS: Computational Spatial Distance to the Start of the Data Cube

VNIR L0 (nm): 507.6, 509.5, 514.5, 520.8, 529.0, 537.4, 545.8, 554.4, 562.7, 574.2, 583.6, 590.4, 598.8, 605.7, 617.5, 630.7, 644.2, 657.2, 670.1, $677.8,691.1,698.4,705.3,711.1,717.9,731.3,738.5,751.5,763.7,778.5,794.0,806.3,819.7,833.7,845.8,859.1,872.8,885.6$

VNIR FWHM (nm): 11.2, 13.6, 19.4, 21.8, 22.6, 20.7, 22.0, 22.2, 22.1, 21.6, 18.0, 19.8, 22.7, 27.8, 29.3, 29.9, 26.9, 30.3, 28.5, 27.8, 30.7, 28.3, $25.4,26.6,27.5,28.2,27.4,27.5,30.5,29.5,25.9,27.3,29.9,28.0,28.9,32.0,30.8,27.9$

VNIR dt to start of the data cube (s): $0.9,0.975,1.05,1.125,1.2,1.275,1.35,1.425,1.5,1.575,1.65,1.725,1.8,0,0.075,0.15,0.225,0.3,0.375$, $0.45,0.525,0.6,0.675,0.75,0.825,0.9,0.975,1.05,1.125,1.2,1.275,1.35,1.425,1.5,1.575,1.65,1.725,1.8$

VNIR ds to start of the data cube $(\mathrm{m}): 3.6,3.9,4.2,4.5,4.8,5.1,5.4,5.7,6,6.3,6.6,6.9,7.2,0,0.3,0.6,0.9,1.2,1.5,1.8,2.1,2.4,2.7,3,3.3,3.6$ $3.9,4.2,4.5,4.8,5.1,5.4,5.7,6,6.3,6.6,6.9,7.2$

SWIR L0 (nm): 1154.1, 1168.6, 1183.7, 1199.2, 1214.3, 1228.2, 1245.7, 1261.2, 1281.7, 1298.6, 1312.9, 1330.7, 1347.2, 1363.2, 1378.7, 1396.7,

$1408.1,1426.3,1438.5,1452.6,1467.0,1479.4,1491.8,1503.8,1516.7,1529.3,1541.6,1553.3,1565.5,1575.5,1581.9,1578.3$

SWIR FWHM (nm): 27.0, 27.0, 26.5, 26.1, 26.7, 27.0, 26.4, 26.3, 26.2, 26.5, 26.6, 25.8, 25.8, 25.6, 26.5, 27.4, 26.9, 28.2, 27.2, 27.1, 28.6, 27.7,

$27.9,27.2,28.8,28.5,28.9,29.8,30.4,27.5,20.5,20.1$

SWIR dt to start of the data cube (s): $0,0.012,0.024,0.036,0.048,0.06,0.072,0.084,0.096,0.108,0.12,0.132,0.144,0.156,0.168,0.18,0.192$, $0.204,0.216,0.228,0.24,0.252,0.264,0.276,0.288,0.3,0.312,0.324,0.336,0.348,0.36,0.372$

SWIR ds to start of the data cube (m): $0,0.05,0.10,0.14,0.19,0.24,0.29,0.34,0.38,0.43,0.48,0.53,0.58,0.62,0.67,0.72,0.77,0.82,0.86,0.91$, $0.96,1.01,1.06,1.10,1.15,1.2,1.25,1.30,1.34,1.39,1.44,1.49$

3) using dense image matching to create a DSM;

4) determination of a radiometric imaging model to transform the digital numbers (DNs) data into reflectance;

5) calculating the hyperspectral image mosaics;

6) estimating surface moisture.

The processing of FPI VNIR images is a well-developed process [15], whereas the FPI SWIR sensor is a new prototype, and the processing required additional development. In the following sections, the geometric $(2,3)$ and radiometric $(1,4,5)$ processing steps and estimation process $(6)$ used in this investigation are described.

\section{Geometric Processing}

Geometric processing determines the image orientations and creates point clouds and DSMs. Because the orientation of each band of the FPI data cube (typically $20-40$ bands) would be computationally heavy, we have developed an approach that determines the orientations of selected reference bands and uses a less demanding band-matching procedure for the rest of the bands [15]. The reference bands are selected so that the temporal range of the images is covered as uniformly as possible. Different subsets of data were processed as follows.

1) Single FPI VNIR camera channel 16 (central wavelength of the band: $\mathrm{L} 0=631 \mathrm{~nm}$; spatial difference to the beginning of the data cube: $\mathrm{ds}=0.6 \mathrm{~m}$ ) was processed to study the geometric performance of single FPI camera band data (118 images).

2) The RGB images and three FPI VNIR camera bands 4 $(\mathrm{L} 0=521 \mathrm{~nm} ; \mathrm{ds}=4.5 \mathrm{~m}), 12(\mathrm{~L} 0=590 \mathrm{~nm} ; \mathrm{ds}=$ $6.9 \mathrm{~m})$, and $16(\mathrm{~L} 0=631 \mathrm{~nm}$; ds $=0.6 \mathrm{~m})$ were simultaneously processed to provide the most accurate orientations for the FPI reference bands (589 images).

3) Five bands of the FPI SWIR camera were simultaneously processed to provide orientations for the SWIR data cubes and SWIR DSM (983 images). The bands were $3(\mathrm{~L} 0=1184 \mathrm{~nm}$; ds $=0.1 \mathrm{~m}), 8(\mathrm{~L} 0=1261 \mathrm{~nm}$; $\mathrm{ds}=0.3 \mathrm{~m}), 11(\mathrm{~L} 0=1313 \mathrm{~nm} ; \mathrm{ds}=0.5 \mathrm{~m}), 24(\mathrm{~L} 0=$ $1504 \mathrm{~nm} ; \mathrm{ds}=1.1 \mathrm{~m})$, and $28(\mathrm{~L} 0=1553 \mathrm{~nm} ; \mathrm{ds}=$ $1.3 \mathrm{~m})$.
4) The RGB images were used to create an accurate DSM that was used as reference for other data sets (235 images).

Agisoft PhotoScan Professional commercial software (AgiSoft LLC, St. Petersburg, Russia) was used to determine the image orientations and to generate dense point clouds over the object area. Its excellent performance has been validated in previous studies [24], [35]. PhotoScan performs photobased 3-D reconstruction using feature detection and dense matching. In each data set, the images were automatically oriented without a priori orientation information. The GPS flight trajectory information could also be used to provide the approximate orientations, which could make the processing faster. In the orientation processing, the PhotoScan quality setting was set to "high"; settings for the number of key points per image were 40000 and those for the final number of tie points per image were 1000; an automated lens calibration was simultaneously performed. According to our experiences, these settings are suitable for the FPI images to provide accurate results and reasonable processing time. An automatic outlier removal was performed using the tools of the software on the basis of the reprojection error $(10 \%$ of points with the largest errors were removed) and reconstruction uncertainty (10\% of points with the largest errors were removed). Finally, some points were manually removed from a sparse cloud, particularly points up in the air or underground. This processing provided the orientation of the images and sparse point clouds in the internal coordinate system of the software.

The object reference coordinate system information was used to transform the image orientations into the desired coordinate system. We used different control data configurations to evaluate if the system has a consistent geometric performance and to study optimal georeferencing configurations: 1) 13 GCPs; 2) positions for images measured by the autopilot's GPS and no GCPs; 3) GPS and one GCP close to the takeoff location; 4) GPS and four GCPs in block corners; 5) GPS and five GCPs (four in block corners and one in the center of the block); 6) four GCPs in block corners; and 7) five GCPs (four in block corners and one in the center of the block). In practical operation, the configurations with a minimum number of GCPs are the most efficient. The standard deviation settings for the GCPs 
were $\sigma_{\mathrm{GCP} X \mathrm{XYZ}}=0.001 \mathrm{~m}$, and for the GPS coordinates, we used a standard deviation of $\sigma_{\mathrm{GPS} X Y Z}=3 \mathrm{~m}$. The projection accuracy was set to 0.1 pixels, and tie point accuracy was set to 4 pixels. The outputs of the final self-calibrating block adjustment were the camera calibrations, and the image exterior orientations and sparse point clouds in the ETRS TM35FIN coordinate system.

In the dense point cloud generation process, for VNIR and SWIR camera images, the full-resolution images were used, and for the RGB point cloud generation, four times downscaled images were used. Depth filtering was used to filter out outliers in the point clouds. For the SWIR data, the PhotoScan quality setting "mild" was used, performing the least filtering. For the VNIR data and for the RGB data, the setting "moderate" was used, assuming a flatter object and to eliminate more height points.

A band-matching procedure was used for the bands that were not included in the orientation processing. Band matching was carried out using a feature-based matching algorithm, and an affine transformation was used to map the bands to the reference bands. In the previous investigations, the accuracy of this approach has been shown to be on the level of a pixel in flat areas [15]. In this matching, the spatial difference to the reference band (derived from ds; Table II) is an important factor impacting the quality of band matching. For the VNIR camera, we used the reference band for each major spectral color range corresponding to the bands that were oriented by PhotoScan: band $4(\mathrm{LO}=521 \mathrm{~nm}$; ds $=4.5 \mathrm{~m})$, band $16(\mathrm{~L} 0=631 \mathrm{~nm}$; $\mathrm{ds}=0.6 \mathrm{~m})$, and band $29(\mathrm{~L} 0=764 \mathrm{~nm}$; $\mathrm{ds}=4.5 \mathrm{~m})$. For the SWIR camera, we used the same five reference bands $(3,8$, 11,24 , and 28) that were oriented in the PhotoScan processing and matched each unoriented band to the temporally closest reference band. For the noisiest bands in the atmospheric absorption region (bands 15-21; L0 1379-1467 nm), we interpolated the orientations from the orientation trajectory determined photogrammetrically in the PhotoScan processing because the matching would not have provided a reliable result.

The geometric accuracy was evaluated by using independent check points and evaluating the DSMs. The 3-D point determination accuracy was assessed using the GCPs that were not included in the georeferencing and VRS-GPS coordinates of the peat sample points as check points. Height accuracy and deformations of the VNIR and SWIR DSMs were assessed by using the RGB DSM as reference. The accuracy of alignment of bands of final image mosaics was evaluated by using an image correlation technique, by matching all the bands to a reference band, and by calculating discrepancies.

\section{E. Radiometric Modeling and Reflectance Mosaic Generation}

Radiometric modeling includes the sensor corrections, the atmospheric correction, correction for the illumination changes and other nonuniformities, and the normalization of illumination and viewing-direction-related nonuniformities by utilizing the bidirectional reflectance distribution function (BRDF) correction.

The sensor corrections for the FPI images include spectral smile correction, photon response nonuniformity correction
(PRNU), and dark signal correction [15], [16]. The PRNU and smile corrections were determined at the laboratory of VTT [16]. The dark signal correction is calculated using a black image collected right before the data capture. In this investigation, all these correction steps were used for the FPI VNIR camera. For the FPI SWIR camera, only the dark signal correction was used; developing laboratory calibration procedures for the prototype sensor was not possible in this investigation. As the SWIR images showed significant sensorrelated nonuniformities, we developed a series of empirical corrections, as described in Section III-E1.

The reflectance transformation was carried out using the empirical line method [40] with the aid of the reflectance panels in the area. For the SWIR images, all panels with nominal reflectance of $0.03,0.10$, and 0.5 were used. For the VNIR images, the brightest panel was not used because it was saturated in most of the bands. The model was

$$
D N=a_{\mathrm{abs}} R e f l+b_{\mathrm{abs}}
$$

where $a_{\mathrm{abs}}$ and $b_{\mathrm{abs}}$ are the parameters for the empirical line model for transforming the reflectance $(R e f l)$ to DN. The transformation was calculated using the image where the panels were the closest to the image center to avoid impacts of reflectance anisotropy in the reference reflectance.

To correct for the atmospheric instability and the impacts of BRDF, a radiometric block adjustment approach was used [15]. The basic principle of the approach is to use the DNs of the radiometric tie points in the overlapping images as observations and to determine the parameters of the radiometric model indirectly via the least squares principle. The model for a DN is

$$
D N=a_{\mathrm{rel} \_j}\left(a_{\mathrm{abs}} R_{\mathrm{jk}}\left(\theta_{i}, \theta_{r}, \varphi\right)+b_{\mathrm{abs}}\right)
$$

where $R_{\mathrm{jk}}\left(\theta_{i}, \theta_{r}, \varphi\right)$ is the bidirectional reflectance factor of the object point, $k$, in image $j ; \theta_{i}$ and $\theta_{r}$ are the illumination and reflected light (observation) zenith angles; $\varphi_{i}$ and $\varphi_{r}$ are the azimuth angles, respectively; $\varphi=\varphi_{r}-\varphi_{i}$ is the relative azimuth angle; and $a_{\mathrm{rel}_{-j}}$ is the relative image-wise correction parameter. The linear BRDF model by Walthall [41] was used to correct the BRDF effects. The estimated nadir reflectance ( $\left.R_{\text {nadir }}\right)$ is

$$
R_{\text {nadir }}=\left(D N / a_{\text {rel_ } j}-b_{\text {abs }}\right) /\left(a_{\text {abs }}\left(a^{\prime} \theta_{r}^{2}+b^{\prime} \theta_{r} \cos \varphi+1\right)\right)
$$

where $\mathrm{a}^{\prime}$ and $\mathrm{b}^{\prime}$ are adjustable BRDF model parameters. Homogeneous distribution of radiometric tie points was generated, with approximately 70 tie points in each image; the DN observation of tie points was calculated in an image window of size $3 \mathrm{~m} \times 3 \mathrm{~m}$ (see details in [15]).

In this investigation, the final image output was a reflectance mosaic. Image mosaics were resampled with a 20-cm GSD from the image block data with the aid of the image orientations and DSM and applying the radiometric model. The reflectance values were taken for each mosaic pixel from the image where the image ray had the smallest difference to the vertical direction. For the VNIR images, we used the full model (4) to calculate reflectance values. We carried out several empirical corrections for the SWIR images (see below), and because 


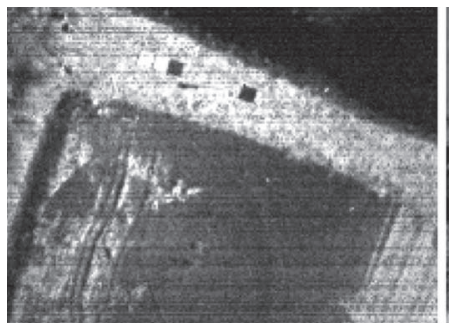

(a)

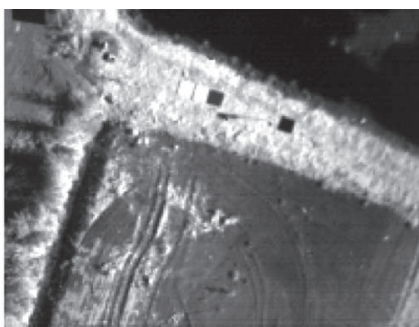

(b)
Fig. 4. Sample images of FPI SWIR bands (a) 21 and (b) 30.

of this, it was feasible to estimate only the relative parameters $\left(a_{\text {rel } \_j}\right)$ in the radiometric block adjustment. It was not possible to solve the BRDF parameters because the empirical corrections strongly correlated with the BRDF effects; thus, default values $a^{\prime}=b^{\prime}=0$ were used. The irradiance observations could be used to calculate the $a_{\text {rel_}} j$ parameters [36], but in this study, the irradiance values were not used.

1) Empirical Radiometric Calibration of the FPI SWIR Camera: The first analysis of the spectra and image mosaics calculated of the FPI SWIR camera images without any corrections indicated that the radiometric calibration was not accurate enough. The mosaics were not homogeneous due to the missing lens falloff calibration. Second, there appeared a decrease in image intensity during the flight and negative DN values. The most probable reason for this distortion was the change in the dark signal during the flight due to sensor cooling. Furthermore, there was a relatively high level of noise in the images (see Fig. 4).

A series of empirical corrections was applied to the images to eliminate these distortions. These corrections were calculated by assuming that the target area had uniform reflectance on average in each spectral band. The wetland area in the northern part of the area was not included in these calculations. All the corrections were calculated separately for each band. To eliminate the impacts of the changes in the dark signal, a strip-wise additive dark current correction was calculated by assuming that the average reflectance of each strip should be the same. The correction was calculated based on the average DNs of each strip in comparison to the average DN of the reference strip (in this case, the first strip). A median-image-based approach was used to eliminate the lens falloff. The assumption was that the median image calculated using all images (except the wetland area) should show uniform intensity, whereas the nonuniformity of the median image indicates systematic radiometric distortions. A multiplicative pixel-wise correction coefficient $L F C(l, m)$ with respect to the central pixel of the median image $\left(\right.$ med_image $\left.\left(\operatorname{row}_{c}, \operatorname{col}_{c}\right)\right)$ was calculated for each pixel $(l, m)$, i.e.,

$$
L F C(l, m)=\text { med_image }\left(\operatorname{row}_{c}, \operatorname{col}_{c}\right) / \operatorname{med} \_i m a g e(l, m) .
$$

The corrected DN is

$$
D N_{\text {cal }}(l, m)=L F C(l, m) D N(l, m) .
$$

\section{F. Remote Sensing of Surface Moisture}

The albedo of peat is known to decrease nonlinearly on wetting, with additional changes in the shape of the spectrum
TABLE III

Statistics of GeOMEtric Processing: Number of IMAges, TiE POINTS, AND PROJECTIONS; REPROJECTION ERROR AND NUMBER of Points and Point Density in Dense Point Cloud

\begin{tabular}{llllll}
\hline \hline Dataset & $\begin{array}{l}\text { N } \\
\text { ima. }\end{array}$ & $\begin{array}{l}\text { N Tie } \\
\text { points }\end{array}$ & N Proj. & $\begin{array}{l}\text { Re- } \\
\text { proj. } \\
\text { Error } \\
\text { (pix) }\end{array}$ & $\begin{array}{l}\text { Dense point } \\
\text { cloud: } \\
\text { N points; } \\
\text { points/m2 }\end{array}$ \\
\hline SWIR & 983 & 61018 & 238888 & 0.323 & $3.8 \mathrm{e} 6 ; 45$ \\
VNIR ch 16 & 118 & 6914 & 37344 & 0.480 & $9.0 \mathrm{e} 6 ; 111$ \\
RGB & 235 & 10264 & 154985 & 0.994 & $13.5 \mathrm{e} 6 ; 103$ \\
RGB + VNIR & 589 & 14538 & 260353 & 0.893 & - \\
\hline \hline
\end{tabular}

particularly near the water absorption features. However, in the range of MCs measured, the changes in albedo could be expected to be approximately linear [31]. We studied the usefulness of spectral features for moisture estimation by calculating linear correlations between the features and moisture and by employing machine learning for the study of nonlinear dependence relations. The data sets used in the study of peat moisture estimation were the VNIR mosaic with BRDF and relative image-wise corrections; the SWIR mosaic with dark signal correction, median-image-based calibration, and relative image-wise corrections; and the RGB mosaic with standard PhotoScan processing scaled to range $0-1$ by dividing the DNs by 255 .

Single-pixel reflectance spectra were collected from the mosaics from the locations corresponding to the collected samples $(20 \mathrm{~cm} \times 20 \mathrm{~cm}$ area); tests using larger sample areas showed reduced correlation with the measured moisture. Reflectance differences $\left(R_{i}-R_{j}\right)$ and ratios $\left(R_{i} / R_{j}\right)$ were calculated for each pair of bands of the concatenated RGB, VNIR, and SWIR spectra of each sample. Linear correlation of the individual bands, band differences, and ratios to the MC was examined using the MATLAB function corr to calculate the Pearson correlation for each set of features. A machine-learning approach based on a support vector machine (SVM) was employed for moisture estimation to take into account possible nonlinearities. A leave-one-out approach was used to estimate the performance of different data sets for the SVM machine learning model generation. The SVM was trained for each set of feature vectors and moistures corresponding to 43 samples and then used to predict the moisture of the remaining sample. The process was repeated for each sample with the individual prediction errors collected together and used to calculate the performance statistics for each data set. SVM training and prediction was performed using $\nu$-SVR (support vector regression) with libSVM [43]. Optimum $\mathrm{C}$ and $\gamma$ parameters for the SVM were determined for a grid of parameters in the $\log _{2} \mathrm{C} \times \log _{2} \gamma$ space, and selecting for each data set the parameters that resulted in the lowest mean square error in the procedure.

\section{RESUlts}

\section{A. Geometric Performance}

Statistics of the geometric processing indicated accurate results (see Table III). The reprojection errors were on the level of $5-7 \mu \mathrm{m}$ in image coordinates for all calculations (about 0.5 pixels for the FPI VNIR, 0.3 pixels for FPI SWIR, and 
TABLE IV

Error Statistics of the Various Geometric Processing Configurations for FPI VNiR IMAges. N GCP: Number OF GCPS; GPS: YES = GPS SUPPORT USED, NO = GPS SUPPORT NOT USED; N CP: NUMBER OF CHECK POINTS

\begin{tabular}{lllllllllll}
\hline \hline N GCP; & N & \multicolumn{2}{l}{ Mean $(\mathrm{m})$} & \multicolumn{3}{c}{ Standard deviation $(\mathrm{m})$} & \multicolumn{3}{c}{ RMSE $(\mathrm{m})$} \\
GPS & $\mathrm{CP}$ & $\mathrm{X}$ & $\mathrm{Y}$ & $\mathrm{Z}$ & $\mathrm{X}$ & $\mathrm{Y}$ & $\mathrm{Z}$ & $\mathrm{X}$ & $\mathrm{Y}$ & $\mathrm{Z}$ \\
\hline 0; yes & 13 & -0.019 & 0.034 & -0.765 & 0.171 & 0.217 & 0.081 & 0.166 & 0.211 & 0.769 \\
1; yes & 12 & 0.473 & 0.279 & -0.436 & 0.297 & 0.344 & 0.376 & 0.552 & 0.432 & 0.566 \\
4; yes & 9 & -0.011 & 0.001 & -0.008 & 0.019 & 0.025 & 0.045 & 0.021 & 0.024 & 0.043 \\
5; yes & 8 & -0.012 & -0.026 & -0.026 & 0.016 & 0.022 & 0.050 & 0.019 & 0.033 & 0.053 \\
4; no & 9 & -0.014 & 0.002 & 0.163 & 0.020 & 0.025 & 0.105 & 0.023 & 0.023 & 0.191 \\
5; no & 8 & -0.014 & -0.024 & -0.031 & 0.016 & 0.021 & 0.057 & 0.021 & 0.032 & 0.061 \\
\hline \hline
\end{tabular}

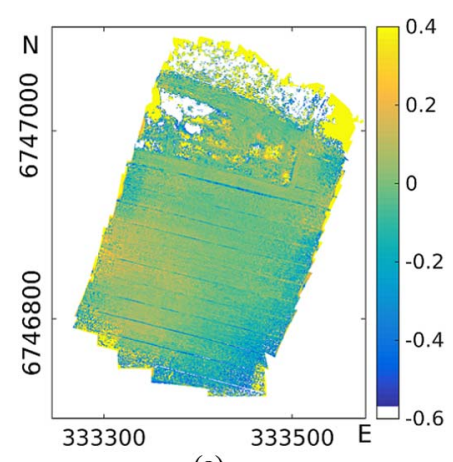

(a)

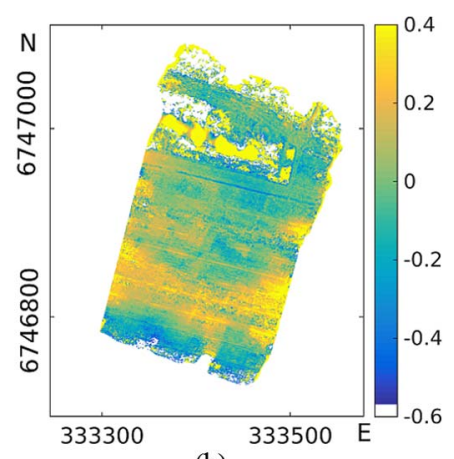

(b)
Fig. 5. Differences to the reference DSM in meters: (a) RGB-VNIR and (b) RGB-SWIR.

one pixel for RGB cameras). The point densities in dense point clouds were about 100 points $/ \mathrm{m}^{2}$ for RGB and FPI VNIR cameras and 45 points $/ \mathrm{m}^{2}$ for FPI SWIR camera. Processing of the SWIR images was less stable than processing of VNIR and RGB cameras, which was due to the poorer block structure, smaller image format, and worse image quality.

The 3-D point determination accuracy of an FPI VNIR data set processed with different georeferencing configurations was studied using the GCPs that were not included in the orientation as check points (see Table IV); the visibility of GCPs was poor in SWIR images because of the larger GSD and the noisier image quality; thus, SWIR images were not analyzed. The best rmse was on the level of $2-3 \mathrm{~cm}$ in the $\mathrm{X}$ and $\mathrm{Y}$ coordinates and $5-6 \mathrm{~cm}$ in height. This accuracy level was obtained when using four GCPs with the autopilot's GPS support or five or more GCPs without the GPS support. The case with the GPS support and no GCPs was of quite low geometric quality, with an rmse of about $0.2 \mathrm{~m}$ in $\mathrm{X}$ and $\mathrm{Y}$ and $0.8 \mathrm{~m}$ in $\mathrm{Z}$.

The results of DSM accuracy assessment are shown in Fig. 5 when using all 13 GCPs in georeferencing and no GPS support; these results represent the best achievable accuracy. The results suggested that the VNIR DSM had a slight tilt (less than $20 \mathrm{~cm}$ ) to the reference surface; the west side was higher than the east side [see Fig. 5(a)]. The SWIR DSM had more deviation from the reference surface with south-northaligned distortion, which was less than $40 \mathrm{~cm}$ [see Fig. 5(b)]. In particular, the VNIR DSM was not significantly deformed in the area surrounded by GCPs. When using the peat sample points as check points, the height rmses were approximately 10-12 cm, at best, for all of the materials (see Table V). The mean errors showed a moderate negative bias of 9-11 cm, which could be due to the difference in measuring the height of the peat surface in the field and from the image. The height standard deviations were on the level of $4 \mathrm{~cm}$ for the RGB and FPI VNIR cameras and about $8 \mathrm{~cm}$ for the FPI SWIR camera. Height rmses were $2-5 \mathrm{~cm}$ for the GCPs, indicating that the DSM fitted very well to these points. One potential explanation for the better height accuracy results with the GCPs is the better measurement accuracy of the well-defined surface of the GCP.

Analysis of the impact of the ground control configuration in the DSM height error is presented in Figs. 6 and 7. In the case of the SWIR data set, the DSM did not show significant deformation [see Figs. 5(b) and 6(f)] when using the configurations with 5 or 13 GCPs and no GPS support; these cases provided also a good height rmse in the peat sample points, on the level of $11 \mathrm{~cm}$ (see Table V). In all cases with the GPS support, the SWIR DSM surface was deformed [see Fig. 6(a)-(e)], and the height rmses were large, $0.35-3 \mathrm{~m}$ in check points (see Table V); the most likely explanation for the poor results is the low quality of the autopilot GPS solution during the SWIR flight. For the VNIR data, the different GCP configurations provided consistent results with the previous analysis when using GCPs as reference (see Tables IV and V and Fig. 5). The DSM did not show deformations in the cases where the rmses were low [see Figs. 5(a) and 7(c), (d), and (f)]; for the cases with poor rmses, the DSMs were deformed [see Fig. 7(a), (b), and (e)].

Assessment of the quality of alignment of individual spectral bands of the mosaics showed good results. For the VNIR data, the mosaic of band 4 was used as reference for all the bands. The discrepancies in the $\mathrm{X}$ and $\mathrm{Y}$ coordinates were less than 1 pixel in $90 \%-99 \%$ of the matched points in bands 1-34 and in $85 \%-90 \%$ of the matched points in bands 35-38. For the SWIR data, mosaics of bands 3 and 28 were used as reference. In most of the bands, the discrepancies were less than 1 pixel in $X$ and $Y$ coordinates in $80 \%-99 \%$ of matched points; in bands $12-14$, the discrepancies were less than 1 pixel in $70 \%-80 \%$ of matched points. In the bands in the atmospheric absorption region for which the orientations were interpolated (bands 15-21), a majority of discrepancies were on the level of 3 pixels and less. These results showed that the individual bands were well aligned. 
TABLE V

Statistics of the DSM Assessment For the VARious Geometric Processing Configurations When USing the 44 INDEPENDENT Check Points (CP) and 13 GCPs as the Reference. Mean, Standard Deviation (STD), and RMSE of Errors $($ ERROR $=$ REFERENCE - INTERPOLATED FROM DSM). N GCP: NUMBER OF GCPS; GPS: YES $=$ GPS USED, $\mathrm{NO}=$ GPS NOT USED

\begin{tabular}{|c|c|c|c|c|c|c|c|}
\hline Data set & $\begin{array}{l}\text { N GCP; } \\
\text { GPS }\end{array}$ & $\operatorname{mean}_{\mathrm{CP}}(\mathrm{m})$ & $\operatorname{std}_{\mathrm{CP}}(\mathrm{m})$ & $\operatorname{RMSE}_{\mathrm{CP}}(\mathrm{m})$ & mean $_{\mathrm{GCP}}(\mathrm{m})$ & $\operatorname{std}_{\mathrm{GCP}}(\mathrm{m})$ & $\operatorname{RMSE}_{\mathrm{GCP}}(\mathrm{m})$ \\
\hline$\overline{\mathrm{RGB}}$ & $13 ;$ no & -0.109 & 0.040 & 0.116 & 0.010 & 0.020 & 0.021 \\
\hline \multirow[t]{7}{*}{ VNIR } & $13 ;$ no & -0.092 & 0.041 & 0.100 & 0.013 & 0.058 & 0.057 \\
\hline & $4 ;$ no & 0.406 & 0.048 & 0.417 & -0.106 & 0.134 & 0.167 \\
\hline & 5 ; no & -0.120 & 0.046 & 0.129 & 0.023 & 0.050 & 0.053 \\
\hline & 0 ; yes & 0.567 & 0.053 & 0.569 & 0.760 & 0.108 & 0.767 \\
\hline & 1 ; yes & 0.412 & 0.151 & 0.438 & 0.394 & 0.370 & 0.531 \\
\hline & 4; yes & -0.140 & 0.049 & 0.148 & 0.009 & 0.054 & 0.053 \\
\hline & 5 ; yes & -0.130 & 0.045 & 0.137 & 0.024 & 0.048 & 0.052 \\
\hline \multirow[t]{7}{*}{ SWIR } & 13 ; no & -0.086 & 0.077 & 0.115 & -0.002 & 0.030 & 0.029 \\
\hline & $4 ;$ no & 0.923 & 0.197 & 0.943 & 0.443 & 0.599 & 0.726 \\
\hline & $5 ;$ no & -0.072 & 0.083 & 0.110 & 0.000 & 0.068 & 0.065 \\
\hline & 0 ; yes & 2.255 & 0.543 & 2.318 & 2.598 & 1.132 & 2.816 \\
\hline & 1 ; yes & -0.043 & 0.526 & 0.522 & 0.363 & 1.370 & 1.365 \\
\hline & 4; yes & -0.310 & 0.216 & 0.376 & 0.039 & 0.386 & 0.373 \\
\hline & 5 ; yes & -0.403 & 0.198 & 0.448 & -0.020 & 0.371 & 0.357 \\
\hline
\end{tabular}

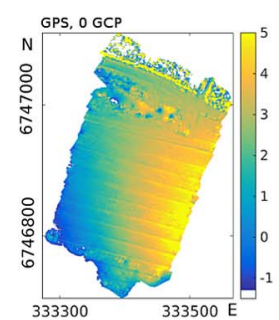

(a)

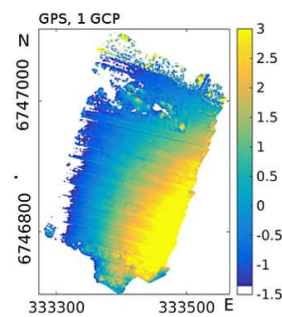

(b)

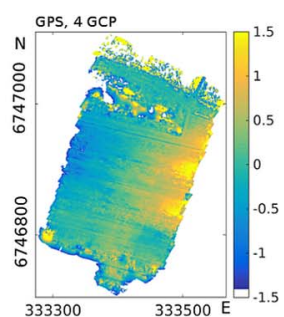

(c)

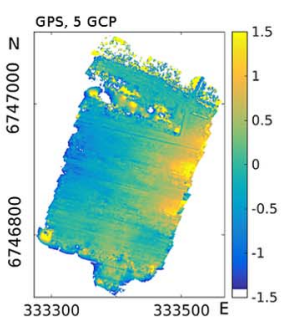

(d)

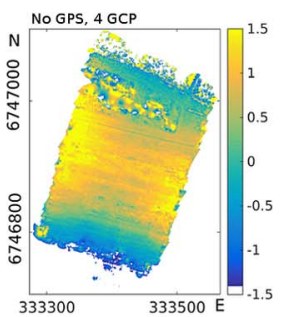

(e)

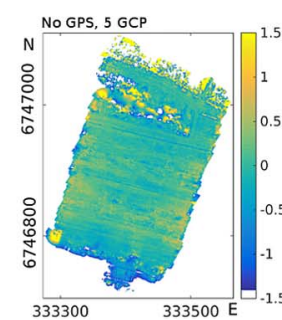

(f)

Fig. 6. Differences (in meters) between the FPI SWIR DSMs and the reference DSM (RGB-SWIR) when using different georeferencing configurations: (a) GPS; (b) GPS, 1 GCP; (c) GPS, 4 GCPs; (d) GPS, 5 GCPs; (e) no GPS, 4 GCPs; (f) no GPS, 5 GCPs.

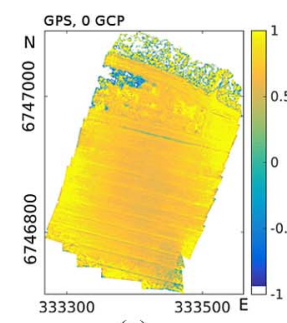

(a)

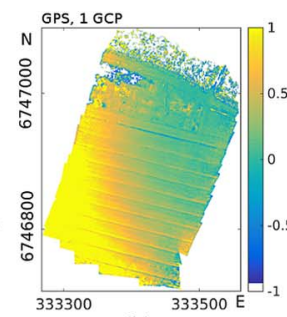

(b)

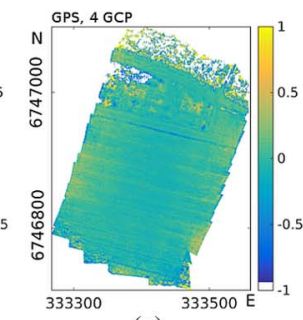

(c)

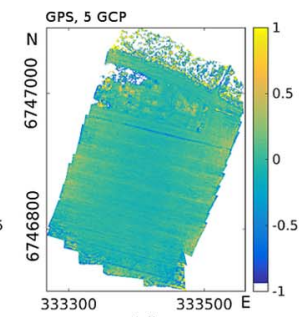

(d)

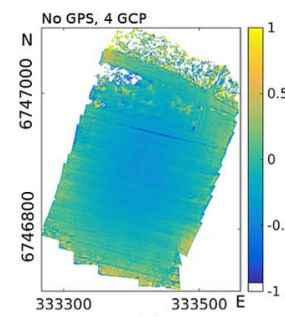

(e)

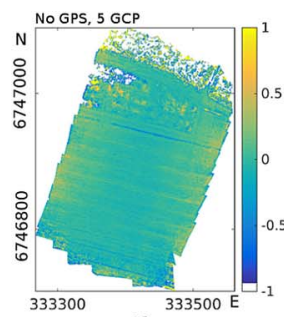

(f)

Fig. 7. Differences (in meters) between the FPI VNIR DSMs and the reference DSM (RGB-VNIR) when using different georeferencing configurations: (a) GPS; (b) GPS, 1 GCP; (c) GPS, 4 GCPs; (d) GPS, 5 GCPs; (e) no GPS, 4 GCPs; (f) no GPS, 5 GCPs.

\section{B. Radiometric Processing}

The incomplete radiometric calibration was visible in the SWIR mosaics without any radiometric corrections (see Fig. 8, left). The dark signal change was visible particularly in band 21 [see Fig. 8(a)]. The dark signal correction and the medianimage-based lens falloff correction compensated for most of the mosaic nonuniformity (see Fig. 8, center). However, some part of the nonuniformity still remained. This could be due to the fact that the strip-wise dark signal correction was not accurate (most likely the dark signal changed continuously due to the temperature changes). After the radiometric block adjustments using the relative image-wise corrections $\left(a_{\text {rel } \_j}\right)$, the mosaics were uniform (see Fig. 8, right) and suitable for the following remote sensing analysis. A three-band SWIR mosaic is shown in Fig. 9(a).

The BRDF correction and the relative image-wise corrections $\left(a_{\text {rel } \_j}\right)$ were used when calculating the VNIR mosaics.
The relative corrections were on the level of $10 \%$. The view angle range in the FPI VNIR images is 0 to $\pm 31^{\circ}$ from the nadir, which is expected to cause BRDF effects in images. The hemispherical directional reflectance factor plot of an area of about $150 \mathrm{~m} \times 150 \mathrm{~m}$ with a point interval of $10 \mathrm{~m}$ indicated that the peat surface was backward scattering with decreasing reflectance toward a forward scattering direction (see Fig. 10). The reflectance anisotropy was about $10 \%$ with a view angle range of 0 to $\pm 25^{\circ}$ and about $20 \%$ from maximum to minimum. The output mosaics of VNIR data had uniform data quality [see Fig. 9(b) and (c)].

\section{Peat Spectra and Moisture Estimation}

The use of spectral information in the assessment of the peat moisture is based on the fact that the more moist the peat is, the darker it is. Some fluctuations appeared in the peat spectra, 


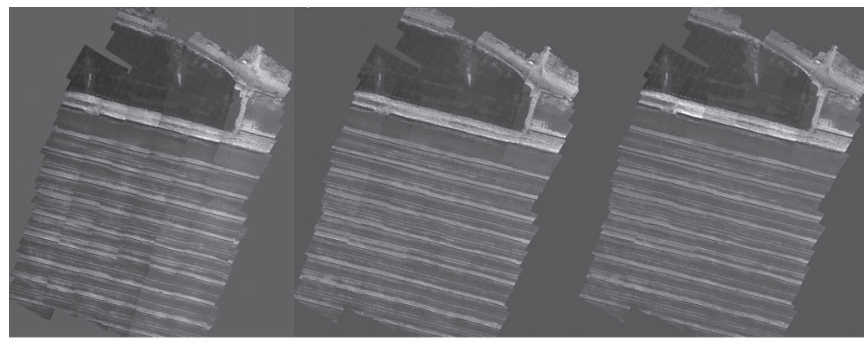

(a)

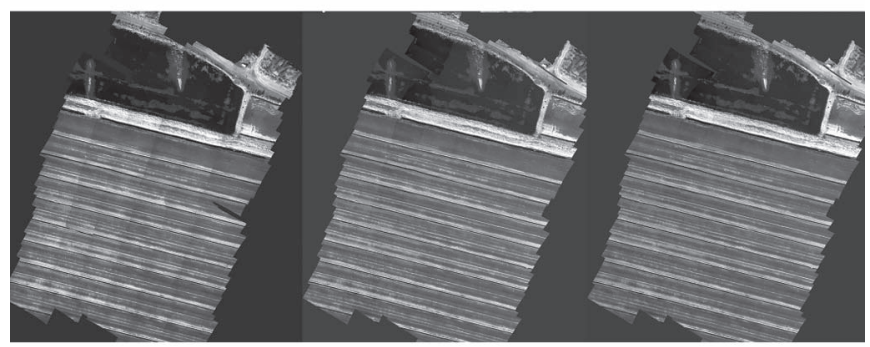

(b)

Fig. 8. Examples of reflectance mosaics with different processing options for SWIR bands (a) 21 and (b) 30. Processing versions from left: original images without corrections; dark signal correction and median image calibration; dark signal correction, median image calibration, and relative correction.

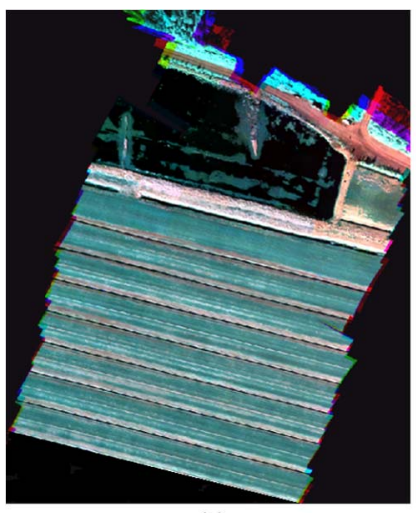

(a)

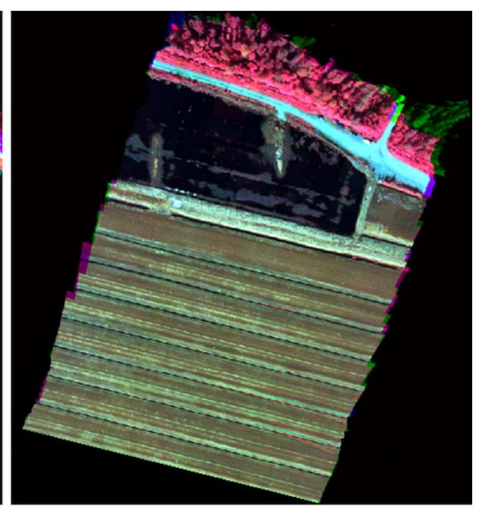

(b)

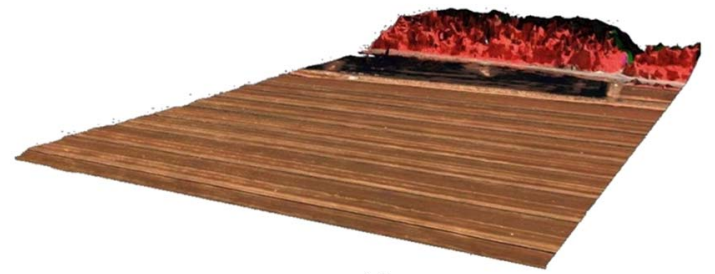

(c)

Fig. 9. Hyperspectral reflectance mosaic from (a) SWIR data (bands at 1184, 1331, and $1553 \mathrm{~nm}$ ) and (b) VNIR data (bands at 520.80, 630.70, and $763.70 \mathrm{~nm}$ ). (c) 3-D perspective visualization of the VNIR mosaic.

but the overall form of the spectra was consistent, showing an increase from green toward NIR wavelengths, a drop at the water absorption region (1350-1450 nm) and a linear increase toward longer wavelengths [see Fig. 11(a)]. Assessment of the reflectance spectra of individual bands with respect to the target moisture indicated correlations of up to 0.63 at highest for SWIR band 7 at $1246 \mathrm{~nm}$; typical correlations were 0.5-0.6 with VNIR and SWIR data sets [see Fig. 11(b)]. For the RGB camera, the correlations were lower, i.e., less than 0.5. The
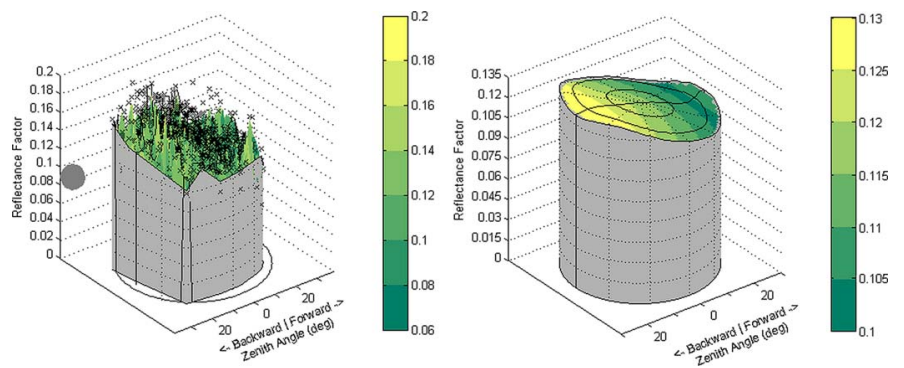

Fig. 10. Reflectance factor observations as the function of the view and illumination angles (left) and fitted BRDF surface (right) for near-infrared band $\mathrm{LO}=764 \mathrm{~nm}$.

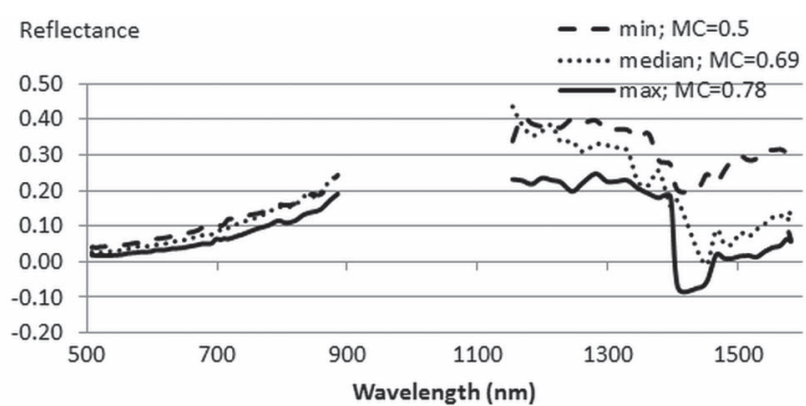

(a)

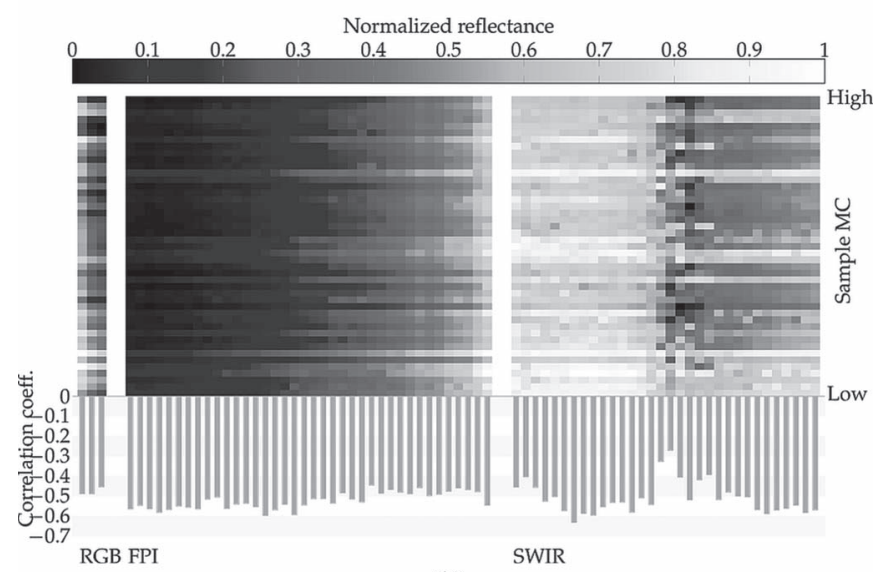

(b)

Fig. 11. (a) Spectra of peat samples with the minimum, maximum, and median MC. (b) Reflectance spectra of all the samples and the correlations of each band with the measured MC for the RGB, VNIR (FPI), and SWIR data sets. The rows and columns correspond, respectively, to different samples and bands. The spectra in each data set are normalized to fill the interval $[0,1]$, and the samples are arranged by their measured MC.

plot shows quite consistent darkening as the MC increased. In cases where correlation is high (such as SWIR band 7), there appeared quite linear change in reflectance. In the water absorption region (1350-1450 nm), the spectra appeared noisy, and the correlations were low.

The correlation coefficients and the associated P-values of the $\mathrm{MC}$ and the reflectance differences $\left(R_{i}-R_{j}\right)$ and ratios $\left(R_{i} / R_{j}\right)$ were collected into square matrices and color coded (see Fig. 12). The highest correlations (with $r=0.66, p<10^{-6}$ ) were obtained using the reflectance difference of SWIR band 7 $(\mathrm{L} 0=1246 \mathrm{~nm})$ and VNIR band $36(\mathrm{~L} 0=859 \mathrm{~nm})$.

Table VI lists the results of the remote sensing analysis of surface moisture by the SVM. The data sets RGB, VNIR, 

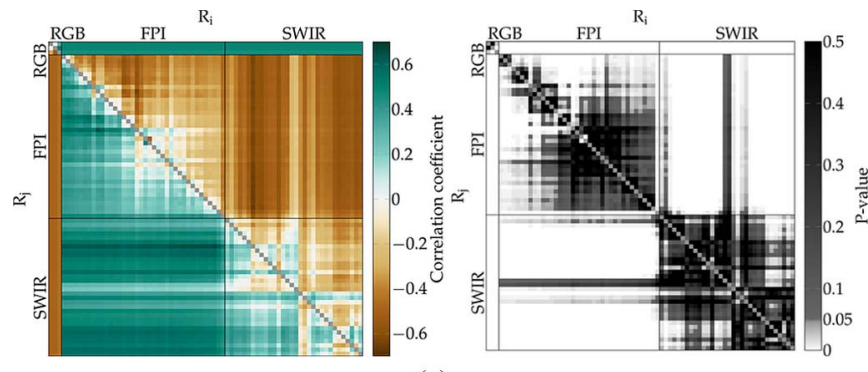

(a)
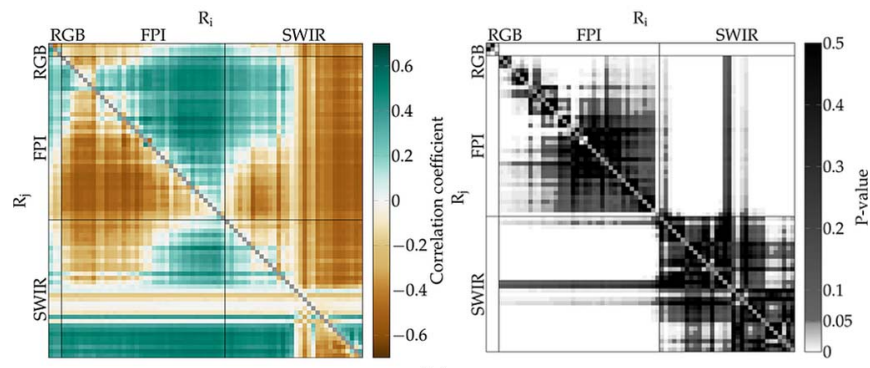

(b)

Fig. 12. Pearson correlation coefficients and associated P-values between the MC and (a) band differences $R_{i}-R_{j}$ and (b) band fractions $R_{i} / R_{j}$ calculated using the RGB, VNIR (FPI), and SWIR data sets. Black lines were added to separate the bands corresponding to different data sets.

TABLE VI

RESUltS OF THE SVM LEAVE-ONE-OUt ANALYSIS. THE ERROR IS COMPUTED FOR EACH SAMPLE AS THE DifFERENCE OF THE REFERENCE AND THE PREDICTED MC (MC $\mathrm{M}_{\mathrm{REF}}-\mathrm{MC}_{\mathrm{PRED}}$; IN PERCENTAGE Points PP). THE STATISTICS INCLUde MEAN ERRor, STANDARD DEVIATION, RMSE, AND NRMSE (RMSE NORMALIZED BY THE MEAN MEASURED MC)

\begin{tabular}{lcccc}
\hline \hline Data & $\begin{array}{c}\text { Mean } \\
(\mathrm{pp})\end{array}$ & $\begin{array}{c}\text { Standard } \\
\text { deviation } \\
(\mathrm{pp})\end{array}$ & $\begin{array}{c}\text { RMSE } \\
(\mathrm{pp})\end{array}$ & $\begin{array}{c}\text { NRMSE } \\
(\%)\end{array}$ \\
\hline RGB+VNIR+SWIR & -0.41 & 5.79 & 5.74 & 8.52 \\
RGB & -1.38 & 6.31 & 6.39 & 9.48 \\
FPI & -0.44 & 5.92 & 5.87 & 8.71 \\
SWIR & -0.25 & 5.74 & 5.68 & 8.44 \\
SWIR7-VNIR36 & 0.16 & 5.21 & 5.15 & 7.65 \\
\hline \hline
\end{tabular}

and SWIR refer to the spectral vectors for each sensor. The RGB + VNIR + SWIR data set is the set of concatenated spectral vectors from the respective data sets. The SWIR7-VNIR36 data set is the vector of reflectance differences of SWIR band $7(\mathrm{~L} 0=1246 \mathrm{~nm})$ and VNIR band $36(\mathrm{~L} 0=859 \mathrm{~nm})$ for each sample. The best accuracy was obtained when using the reflectance difference of SWIR band 7 and VNIR band 36, which gave the rmse of $5.21 \mathrm{pp}$ (pp is the mass fraction in percentage points) and a normalized rmse (nrmse) of $7.61 \%$. When using individual cameras, the rmses were $6.39,5.87$, and $5.68 \mathrm{pp}$, and the nrmses were $9.48 \%, 8.71 \%$, and $8.44 \%$ for the RGB, VNIR, and SWIR cameras, respectively.

\section{DISCUSSION}

This investigation studied the performance of two novel lightweight frame format hyperspectral cameras onboard a small UAV in measuring the 3-D surface model and surface moisture of a peat production area. The VNIR FPI camera was shown to be operational in previous studies [15], [32]-[35]. The SWIR FPI camera [19] was a completely new prototype camera. The UAV system was also equipped with a high-spatialresolution customer RGB camera. Data sets were captured over a peat production area using a flying height of 90-94 m with GSDs of 2.5, 9.5, and $15 \mathrm{~cm}$ for the RGB, VNIR, and SWIR cameras, respectively.

\section{A. Considerations on Geometric Performance}

As the FPI-based imager captures spectral data cubes using the time-sequential principle, the individual bands of the data cubes are unregistered. We used a methodology where several bands of the set of unregistered data cubes were simultaneously oriented in the self-calibrating bundle block adjustment. The success of this approach is dependent on the invariance of the matching algorithm to the spectral differences in images. The commercial Agisoft PhotoScan software was quite tolerable to the spectral differences within visible range $(400-650 \mathrm{~nm})$ and SWIR range $(1100-1600 \mathrm{~nm})$ and provided accurate integrated orientation result. The procedure is based on interest points, which are stable under viewpoint and lighting variations and their descriptors based on each points' local neighborhood. Other software programs have similar algorithms [24], and they are expected to be functional as well; however, each method needs to be confirmed. The use of several bands in the processing simultaneously made the processing more robust by providing better overlaps between the images of the block. The bands that were not included in the block adjustment were successfully registered to the oriented bands by a band-matching process. A majority of the discrepancies between the bands were one pixel or less; these results were consistent with previous studies performed with the VNIR camera in areas with flat topography [15].

We validated the geometric performance of the FPI cameras by using different sets of ground reference data, including variable configurations of GCPs and autopilots' GPS observations. The detailed analysis using the accurate targeted XYZ control points was possible for VNIR imagery. The best results were on the level of $2-3 \mathrm{~cm}$ in $X$ and $Y$ coordinates and $5-6 \mathrm{~cm}$ in height. The cases based only on the autopilot's GPS data showed quite low geometric accuracy, which is in line with expected quality when using navigation-grade GPS reference [44]. The best height accuracy levels were approximately $10-12 \mathrm{~cm}$ for all of the data sets in the independent check points located in the swamp surface. This estimate included the uncertainty of peat surface measurement and the quality of the measurement method.

The geometric performance of the SWIR data set was worse than that of other cameras, which was due to the poorer block structure (lower image and strip overlaps), potentially lower accuracy of the autopilot's GPS data, smaller image size, and lower image quality. In this investigation, the block structure was optimized for the SWIR camera, flat object, and mosaic calculation; thus, the overlap of $40 \%-45 \%$ was used between the image strips; as the flight trajectory was the same for the RGB and VNIR cameras that had larger FOVs, the resulting block geometry was better for those data sets. The assessment 
showed that the FPI-based sensors were capable of providing good DSMs. When concerning with the geometric performance of the new sensors, the potential deficiency with hyperspectral cameras in comparison to good-quality customer digital color cameras is the smaller number of pixels (poorer spatial resolution), smaller image size, and lower signal-to-noise ratio (because of measuring narrower spectral bands). Furthermore, in the SWIR range, the decreasing level of solar illumination sets additional demands on the sensor when a high signalto-noise ratio is anticipated [42]. In the end, the propagation of errors will follow the photogrammetric theories [45]. Most importantly, the block structure (overlaps, cross-strips, GCPs) and the quality of the direct orientation observations impact the accuracy of orientations, and the level of signal-to-noise ratio impacts the quality of matching [22], [46]. To obtain high DSM quality, the overlaps within and between the strips should be at least $80 \%$ and $60 \%$, respectively, and crossing flight strips are advantageous. In the practical operation, the recommended approach is to integrate a high-spatial-resolution RGB camera with a hyperspectral sensor, to obtain the most accurate DSM and desired spectral properties, if this is possible with the UAV platform in use.

\section{B. Aspects of Spectral Measurement Quality}

The new SWIR prototype had some shortcomings in the spectral measurement quality, which will be improved in the next versions of the camera. These included the missing PRNU calibration as well as the change in the dark signal during the flight. For the miniaturized sensors, temperature calibration together with a correction algorithm based on a model of the sensor behavior is likely the best approach to eliminate temperature effects, since stabilization of sensor temperature might be challenging due to the weight limitations. Several other researchers have also pointed out the importance of calibrating small hyper- and multi-spectral cameras accurately [7], [9], [14], [34], [47]. Dark signal correction based on the dark image collected before the flight appeared to be inaccurate because of the changes in the sensor temperature during the flight. A simple improvement to this procedure would be to capture the dark image before and after the campaign. More rigorous approaches would be determining the temperature impacts on the dark image in controlled conditions or integrating dark signal measurement to the data capture process. The empirical processing steps tailored for this data set were capable of compensating for these limitations and provided suitable data quality for further processes. The radiometric block adjustment approach was functional in eliminating the remaining radiometric nonuniformities in the image mosaics [15]. The accurate radiometric processing in difficult conditions will be an important step in the UAV-based hyperspectral remote sensing. In the future, the radiometric quantities in UAV remote sensing need to be carefully considered [42], [48], for example, are the images processed to reflectance or some other quantities and are the anisotropic reflectance effects compensated for. Furthermore, comprehensive studies concerning the performance of different radiometric correction approaches should be carried out.
In comparison to conventional pushbroom technology, the interesting feature of the frame format hyperspectral cameras is the possibility to collect hyperspectral image blocks with stereoscopic multiview overlaps. On the other hand, for the first-generation frame format hyperspectral cameras, the spectral resolution is still poorer (FWHM on the level of 10-30 nm), and the number of spectral bands is lower (20-40) than that of more mature pushbroom techniques, which typically provide hundreds of spectral bands with FWHMs of 2-10 nm [7]-[11]. The spectral performance of the FPI cameras is expected to improve in future systems, and the latest commercial cameras already offer improved performance (www.rikola.fi).

\section{Remote Sensing of Surface Moisture}

We evaluated the performance of UAV remote sensing with the RGB camera and the FPI-based VNIR and SWIR cameras in the measurement of surface moisture of a peat production area. The results indicated good agreement of the reflectance signatures in images with the moisture of the object. The peat moisture estimation was more accurate with the FPI SWIR camera than with the FPI VNIR or traditional RGB camera. The best accuracy was obtained when using the reflectance difference of SWIR band $7(\mathrm{~L} 0=1246 \mathrm{~nm})$ and VNIR band $36(\mathrm{~L} 0=859 \mathrm{~nm})$, which gave an rmse of $5.21 \mathrm{pp}$ and an nrmse of $7.61 \%$. Based on our experiences in using a series of peat samples with controlled moisture levels in a laboratory setting, higher accuracy moisture estimation from the spectra should be possible. In particular, the low reflectance and the noisiness of the UAV SWIR spectra near the main water absorption at 1400-nm feature limited the accuracy of the estimation.

Here, the training of the SVM was based on samples from the imaged mire. Due to the nonlinear effects of humification and the differences in the chemical composition of the peat [31], [49], it is not expected that an empirical model trained on one mire is generalizable to other mires. The next steps in research would be gathering a series of training material from different humification levels of peat and performing laboratory testing to separate the effects of humification and moisture on the spectra. To summarize, the major uncertainty components in our experiment included sample gathering, accuracy of positioning, accuracy of moisture measurement system, and humification level. When these factors are well controlled, we assume that the general accuracy of estimation is higher.

The presented technology allows many further improvements. We did not utilize the gathered 3-D geometry in the moisture estimation; it is expected that the topographic information would support the estimation of the surface and belowsurface moisture in the peat mire. We used the most nadir approach in the analysis of the reflectance data, but in the future, the multiview information should be used more efficiently. The potential of multidirectional observations (BRDF) and a physical-radiative-transfer-based approach in estimating the MC needs further study, to develop general methods that do not require in situ reference. The future autonomous UAV systems are feasible for repetitive data acquisition and multitemporal analyses, which might improve the estimation accuracy levels. 
Moreover, the potential of other spectral ranges, particularly multitemporal thermal imaging, should be studied [29], [30].

\section{Outlook}

Based on the results, the FPI hyperspectral technology follows the principles of central perspective imaging, thus enabling the utilization of latest innovations in mainstream computer vision and photogrammetric technologies in developing highly automatic and autonomous applications [1], [2], [20]-[26]. In this investigation, the geometric, radiometric, and peat surface moisture reference were based on field measurements. It will be important to develop reliable methods that do not require interactive field measurements to allow automatic and autonomous operation in the future. These methods will require accurate geometric and radiometric calibration of sensors [9], [14], [34], accurate satellite positioning methods [2], [25], automatic radiometric correction, and detailed understanding of the remote sensing task. When small differences in object characteristics, such as the $\mathrm{MC}$, are of interest, the requirements for the spectral and geometric quality of the remote sensing data are high. This means that the spectral and topographic information has to be accurate and unbiased; the detailed requirements for the data quality need to be studied in the future investigations.

Peat production areas form an environmental risk factor due to the threat of self-ignition. Furthermore, efficiency of peat production could be improved if using efficient remote-sensingbased techniques in estimating optimal time for harvesting and managing the area. Our results indicated that the UAV techniques could be an efficient tool for further optimizing and monitoring of the environmental impacts of peat production. In addition to monitoring peat production areas, the presented approach can be applied for any other application requiring repetitive monitoring in relatively flat surfaces, such as in precision agriculture [15]. The technology has also been demonstrated in large areas (several $\mathrm{km}^{2}$ ) (unpublished results) and in a complex 3-D environment in forest [35]; the technology is also suitable in these applications, but in complex environments, a more complicated overall solution is required.

\section{CONCLUSION}

This paper has studied the performance of two novel lightweight FPI-based hyperspectral frame format cameras in measuring the 3-D surface model and surface moisture of a peat production area. The SWIR range camera was a new prototype, whereas the visible to near-infrared range camera was more mature technology. Moreover, a high-spatial-resolution consumer RGB camera was used. More rigid image geometry as well as the possibility for stereoscopic measurements and multiple object views are important advantages of the frame format geometry in comparison to conventional hyperspectral imaging technology based on pushbroom geometry.

The results were promising, indicating that UAV-based remote sensing could significantly improve the efficiency and the environmental safety aspects of peat production. In addition to monitoring peat production areas, the technology is functional in various remote sensing applications. As the FPI technology follows the theories of central perspective imaging, it is also well suited for developing automatic and autonomous applications utilizing the latest innovations in photogrammetry and computer vision.

\section{REFERENCES}

[1] C. Zecha, J. Link, and W. Claupein, "Mobile sensor platforms: Categorisation and research applications in precision farming," J. Sens. Sens. Syst., vol. 2, no. 1, pp. 51-72, 2013.

[2] I. Colomina and P. Molina, "Unmanned aerial systems for photogrammetry and remote sensing: A review," ISPRS J. Photogramm. Remote Sens., vol. 92, pp. 79-97, Jun. 2014.

[3] E. Hunt, Jr. et al., "Acquisition of NIR-green-blue digital photographs from unmanned aircraft for crop monitoring," Remote Sens., vol. 2, no. 1, pp. 290-305, 2010

[4] C. Lelong, "Assessment of unmanned aerial vehicles imagery for quantitative monitoring of wheat crop in small plots," Sensors, vol. 8, no. 5, pp. 3557-3585, 2008.

[5] G. Zhou, "Near real-time orthorectification and mosaic of small UAV video flow for time-critical event response," IEEE Trans. Geosci. Remote Sens., vol. 47, no. 3, pp. 739-747, Mar. 2009.

[6] A. Goetz, "Three decades of hyperspectral remote sensing of the Earth: A personal view," Remote Sens. Environ., vol. 113, pp. S5-S16, Sep. 2009.

[7] P. Zarco-Tejada, V. Gonzalez-Dugo, and J. Berni, "Fluorescence, temperature and narrow-band indices acquired from a UAV platform for water stress detection using a micro-hyperspectral imager and a thermal camera," Remote Sens. Environ., vol. 117, pp. 322-337, Feb. 2012.

[8] R. Hruska, J. Mitchell, M. Anderson, and N. Glenn, "Radiometric and geometric analysis of hyperspectral imagery acquired from an unmanned aerial vehicle," Remote Sens., vol. 4, no. 12, pp. 2736-2752, 2012.

[9] A. Büttner and H. Röser, "Hyperspectral remote sensing with the UAS 'Stuttgarter Adler'-System setup, calibration and first results," Photogrammetrie-Fernerkundung-Geoinform., vol. 2014, no. 4, pp. 265-274, 2014.

[10] A. Lucieer, Z. Malenovsky, T. Veness, and L. Wallace, "HyperUASimaging spectroscopy from a multirotor unmanned aircraft system," J. Field Robot., vol. 31, no. 4, pp. 571-590, Jul./Aug. 2014.

[11] J. Suomalainen et al., "A lightweight hyperspectral mapping system and photogrammetric processing chain for unmanned aerial vehicles," Remote Sens., vol. 6, no. 11, pp. $11013-11030,2014$.

[12] K. Uto, H. Seki, G. Saito, and Y. Kosugi, "Characterization of rice paddies by a UAV-mounted miniature hyperspectral sensor system," IEEE J. Sel. Topics Appl. Earth Observ. Remote Sens., vol. 6, no. 2, pp. 851-860, Apr. 2013.

[13] A. Burkart et al., "Angular dependency of hyperspectral measurements over wheat characterized by a novel UAV based goniometer," Remote Sens., vol. 7, no. 1, pp. 725-746, 2015

[14] H. Aasen, A. Burkart, A. Bolten, and G. Bareth, "Generating 3-D hyperspectral information with lightweight UAV snapshot cameras for vegetation monitoring: From camera calibration to quality assurance," ISPRS J. Photogramm. Remote Sens., vol. 108, pp. 245-259, Oct. 2015.

[15] E. Honkavaara et al., "Processing and assessment of spectrometric, stereoscopic imagery collected using a lightweight UAV spectral camera for precision agriculture," Remote Sens., vol. 5, no. 10, pp. 5006-5039, 2013.

[16] J. Mäkynen et al., "Unmanned Aerial Vehicle (UAV) operated megapixel spectral camera," in Proc. SPIE Electro-Opt. Remote Sens., Photon. Technol., Appl. V, 2011, vol. 8369, pp. 1-9, doi: 10.1117/12.897712.

[17] H. Saari et al., "Unmanned Aerial Vehicle (UAV) operated spectral camera system for forest and agriculture applications," in Proc. SPIE Remote Sens. Agriculture, Ecosyst., Hydrol. XIII, 2011, vol. 8174, pp. 1-15, doi: $10.1117 / 12.897585$.

[18] H. Saari et al., "Miniaturized hyperspectral imager calibration and UAV flight campaigns," in Proc. SPIE Sens., Syst., Next-Gener. Satell. XVII, 2013, vol. 8889, pp. 1-12, doi: 10.1117/12.2028972.

[19] R. Mannila, C. Holmlund, H. Ojanen, A. Näsilä, and H. Saari, "ShortWave Infrared (SWIR) spectral imager based on Fabry-Pérot interferometer for remote sensing," in Proc. SPIE Sens., Syst., Next-Gener. Satell. XVIII, 2014, vol. 9241, pp. 1-8, doi: 10.1117/12.2067206.

[20] C. Wu, "Towards linear-time incremental structure from motion," in Proc. Int. Conf. 3DV , 2013, pp. 127-134, doi: 10.1109/3DV.2013.

[21] F. Leberl et al., "Point clouds: LIDAR versus 3-D vision," Photogramm. Eng. Remote Sens., vol. 76, no. 10, pp. 1123-1134, 2010. 
[22] T. Rosnell and E. Honkavaara, "Point cloud generation from aerial image data acquired by a quadrocopter type micro unmanned aerial vehicle and a digital still camera," Sensors, vol. 12, no. 12, pp. 453-480, 2012.

[23] J. Lisein, M. Pierrot-Deseilligny, S. Bonnet, and P. Lejeune, "A photogrammetric workflow for the creation of a forest canopy height model from small unmanned aerial system imagery," Forests, vol. 4, no. 4, pp. 922-944, 2013.

[24] A. Eltner and D. Schneider, "Analysis of different methods for 3-D reconstruction of natural surfaces from parallel-axes UAV images," Photogramm. Rec., vol. 30, no. 151, pp. 279-299, Sep. 2015.

[25] B. Jagt, A. Lucieer, L. Wallace, D. Turner, and M. Durand, "Snow depth retrieval with UAS using photogrammetric techniques," Geosciences, vol. 5, no. 3, pp. 264-285, 2015.

[26] S. Puliti, H. Olerka, T. Gobakken, and E. Nässet, "Inventory of small forest areas using an unmanned aerial system," Remote Sens., vol. 7, no. 8, pp. 9632-9654, 2015.

[27] G. P. Petropoulos, G. Ireland, and B. Barrett, "Surface soil moisture retrievals from remote sensing: Current status, products \& future trends," Phys. Chem. Earth, A/B/C, vol. 83/84, pp. 36-56, 2015.

[28] L. K. DeBell, K. Anderson, R. E. Brazier, N. King, and L. Jones, "Water resource management at catchment scales using lightweight UAVs: Current capabilities and future perspectives," J. Unmanned Veh. Syst., vol. 3 , pp. 1-24, 2015, doi: 10.1139/juvs-2015-0026.

[29] D. J. Luscombe, K. Anderson, N. Gatis, E. Grand-Clement, and R. E. Brazier, "Using airborne thermal imaging data to measure nearsurface hydrology in upland ecosystems," Hydrol. Process., vol. 29, no. 6, pp. 1656-1668, Mar. 2015, doi: 10.1002/hyp.10285.

[30] M. Tervo, E. Kiukaanniemi, and T. Kauppinen, "Applications of aerial thermography in peat production in Finland," in Proc. SPIE Thermosense XV, Int. Conf. Thermal Sens. Imaging Diagnostic Appl., Apr. 6, 1993, vol. 101, pp. 1-9, doi: 10.1117/12.141959.

[31] J. McMorrow, A. Al-Roichdi, M. Evans, and M. Cutler, "The effect of moisture content and humification on the hyperspectral reflectance of peat," in Proc. RSPSoc, Scales Dyn. Observ. Environ., Nottingham, U.K., 2003, pp. 1-11.

[32] J. Kaivosoja et al., "A case study of a precision fertilizer application task generation for wheat based on classified hyperspectral data from UAV combined with farm history data," in Proc. SPIE Remote Sens. Agriculture, Ecosyst., Hydrol. XV, Dresden, Germany, Sep. 23-26, 2013, pp. 1-9.

[33] I. Pölönen, H. Saari, J. Kaivosoja, E. Honkavaara, and L. Pesonen, "Hyperspectral imaging based biomass and nitrogen content estimations from light-weight UAV," in Proc. SPIE Remote Sens. Agriculture, Ecosyst., Hydrol. XV, 2013, vol. 8887, pp. 1-9, doi: 10.1117/12.2028624.

[34] G. Bareth et al., "Low-weight and UAV-based hyperspectral fullframe cameras for monitoring crops: Spectral comparison with portable spectroradiometer measurements," Photogrammetrie-FernerkundungGeoinform., vol. 2015, no. 1, pp. 69-79, 2015.

[35] R. Näsi et al., "Using UAV-based photogrammetry and hyperspectral imaging for mapping bark beetle damage at tree-level," Remote Sens., vol. 7, no. 11, pp. 15 467-15493, 2015.

[36] T. Hakala et al., "Spectral imaging from UAVs under varying illumination conditions," Int. Archives Photogramm. Remote Sens. Spatial Inf. Sci., vol. XL-1/W2, pp. 189-194, 2013.

[37] P. Häkli, "Practical test on accuracy and usability of virtual reference station method in finland," in FIG Working Week. Athens, Greece: The Olympic Spirit in Surveying, May 22-27, 2004.

[38] E. Honkavaara, L. Markelin, T. Hakala, and J. Peltoniemi, "The metrology of directional, spectral reflectance factor measurements based on area format imaging by UAVs," Photogrammetrie-FernerkundungGeoinform., vol. 2014, no. 3, pp. 175-188, Jun. 2014.

[39] J. I. Peltoniemi et al., "Technical notes: A detailed study for the provision of measurement uncertainty and traceability for goniospectrometers," J. Quant. Spectrosc. Radiat. Transf., vol, 146, pp. 376-390, Oct. 2014.

[40] G. Smith and E. Milton, "The use of the empirical line method to calibrate remotely sensed data to reflectance," Int. J. Remote Sens., vol. 20, no. 13, pp. 2653-2662, 1999.

[41] C. Walthall, J. Norman, J. Welles, G. Campbell, and B. Blad, "Simple equation to approximate the bidirectional reflectance from vegetative canopies and bare soil surfaces," Appl. Opt., vol. 24, no. 3, pp. 383-387, 1985.

[42] J. Schott, Remote Sensing. New York, NY, USA: Oxford Univ. Press, 2007.

[43] C. Chang and C. Lin, "LIBSVM," ACM Trans. Intell. Syst. Technol., vol. 2, no. 3, pp. 1-27, Apr. 2011.

[44] D. Turner, A. Lucieer, and C. Watson, "An automated technique for generating georectified mosaics from ultra-high resolution Unmanned
Aerial Vehicle (UAV) imagery, based on Structure from Motion (SfM) point clouds," Remote Sens., vol. 4, no. 5, pp. 1392-1410, 2012.

[45] K. Kraus and P. Waldhausl, Photogrammetry. Bonn, Germany: Dummler, 1993.

[46] E. Honkavaara, L. Markelin, T. Rosnell, and K. Nurminen, "Influence of solar elevation in radiometric and geometric performance of multispectral photogrammetry," ISPRS J. Photogramm. Remote Sens., vol. 67, no. 1, pp. 13-26, Jan. 2012.

[47] J. Kelcey and A. Lucieer, "Sensor correction of a 6-band multispectral imaging sensor for UAV remote sensing," Remote Sens., vol. 4, no. 5, pp. 1462-1493, 2012.

[48] G. Schaepman-Strub, M. Schaepman, T. Painter, S. Dangel, and J. Martonchik, "Reflectance quantities in optical remote sensingDefinitions and case studies," Remote Sens. Environ., vol. 103, no. 1, pp. 27-42, 2006.

[49] D. J. M. Hayes, M. H. B. Hayes, and J. J. Leahy, "Analysis of the lignocellulosic components of peat samples with development of near infrared spectroscopy models for rapid quantitative predictions," Fuel, vol. 150, pp. 261-268, Jun. 2015.

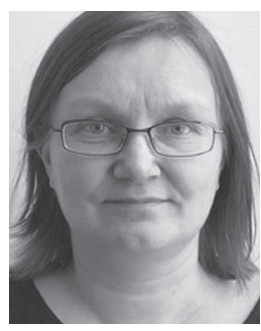

Eija Honkavaara received the D.Sc. degree in technology in photogrammetry from the Helsinki University of Technology, Espoo, Finland, in 2008.

She is currently a Research Manager and a Research Group Leader with the Department of Remote Sensing and Photogrammetry, Finnish Geospatial Research Institute, Masala, Finland. Her research interests include photogrammetry, unmanned aerial vehicles, radiometric and geometric calibration, computer vision, and hyperspectral environmental monitoring applications.

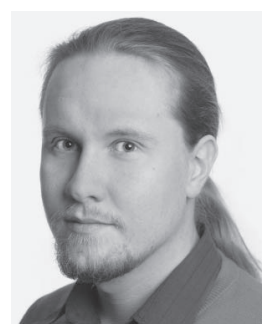

Matti A. Eskelinen received the M.Sc. degree in theoretical physics from the University of Jyväskylä (JYU), Jyväskylä, Finland, in 2015. He is currently working toward the Ph.D. degree in computational hyperspectral analysis with the Department of Mathematical Information Technology, JYU.

His research interests include hyperspectral image analysis, inverse problems, machine learning, and geometric algebra.

Mr. Eskelinen is currently the Vice Chairman of the Jyväskylä Physical Society.

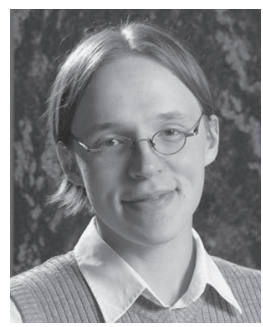

Ilkka Pölönen received the Ph.D. degree in information technology from the University of Jyväskylä, Jyväskylä, Finland.

$\mathrm{He}$ is the Head of the Spectral Imaging Laboratory with the Department of Mathematical Information Technology, University of Jyväskylä. His research interests include data analysis, mathematical modeling, and numerical simulations.

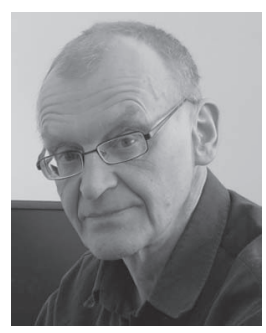

Heikki Saari received the D.Sc. degree in technology in technical physics from Helsinki University of Technology, Espoo, Finland, in 1996.

$\mathrm{He}$ is a Principal Scientist with the Microelectronic Systems Group, VTT Technical Research Centre of Finland, Espoo. He is the author or coauthor of more than 100 publications and 12 patents. His current research interests include microopto-electro-mechanical system spectrometers and Fabry-Pérot interferometer-based hyperspectral imagers in unmanned aerial vehicle remote sensing, medical, space, and industrial applications. 
Harri Ojanen received the Ph.D. degree in mathematics from Rutgers University, New Brunswick, NJ, USA, in 1999.

He is currently with the Microspectrometers Group, VTT Technical Research Centre of Finland, Espoo, Finland. His research interests include the application of miniature framing imaging spectrometer systems based on Fabry-Pérot interferometers into environmental monitoring and healthcare.

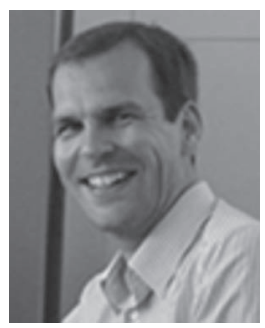

Rami Mannila received the Lic.Sc. degree in physics from the University of Helsinki, Helsinki, Finland, in 2009.

$\mathrm{He}$ is a Senior Scientist with the VTT Technical Research Centre of Finland, Espoo, Finland. His research interests include microspectrometers, unmanned aerial vehicles, hyperspectral imagers, and spectrometer-based environmental monitoring applications.

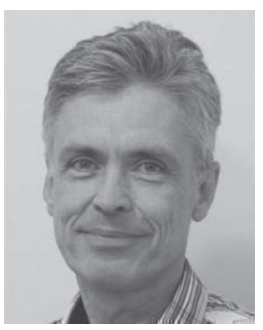

Christer Holmlund received the Master's degree in applied electronics and the Lic.Tech. degree in measurement technology from Helsinki University of Technology, Espoo, Finland, in 1983 and 1990, respectively.

He is a Senior Scientist with the VTT Technical Research Centre of Finland, Espoo. His research interests include electronics and firmware design of microspectrometers and hyperspectral imagers.

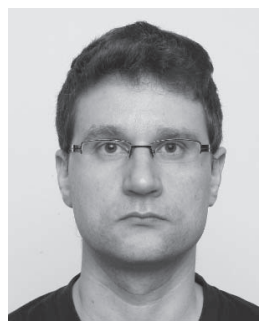

Teemu Hakala received the M.Sc. degree in technology in electronics from Helsinki University of Technology, Espoo, Finland, in 2009. He is currently working toward the Ph.D. degree with Aalto University, Espoo.

He is a Research Scientist with the Department of Remote Sensing and Photogrammetry, Finnish Geospatial Research Institute, Masala, Finland. His research interests include unmanned aerial vehicle sensor technology, radiometric measurements, hyperspectral environmental monitoring, hyperspectral

Paula Litkey received the M.Sc. and Lic.Tech. degrees in electrical engineering from Helsinki University of Technology, Espoo, Finland, in 1998 and 2004, respectively.

She is a Senior Research Scientist with the Department of Remote Sensing and Photogrammetry, Finnish Geospatial Research Institute, Masala, Finland. Her research interests include point cloud applications, surface models, and change detection.

Tomi Rosnell received the M.Sc. degree in technology in photogrammetry from the Helsinki University of Technology, Espoo, Finland, in 2010.

$\mathrm{He}$ is a Research Scientist with the Department of Remote Sensing and Photogrammetry, Finnish Geospatial Research Institute, Masala, Finland. His current research interests include indoor and outdoor close-range photogrammetry, three-dimensional modeling, and unmanned aerial vehicles.

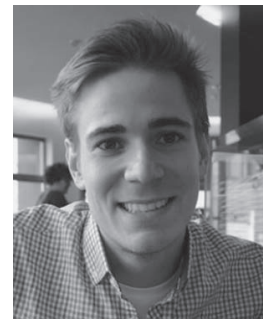

applications.

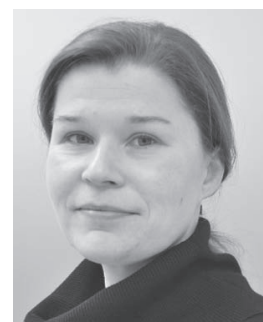

Niko Viljanen received the B.Sc. degree in technology in geodesy and photogrammetry from Aalto University, Espoo, Finland, in 2016, where he is currently working toward the M.Sc. degree in technology in photogrammetry and remote sensing.

$\mathrm{He}$ is a Research Assistant with the Department of Remote Sensing and Photogrammetry, Finnish Geospatial Research Institute, Masala, Finland. His research interests include photogrammetry, unmanned aerial vehicles, geometric calibration, and hyperspectral environmental monitoring

Merja Pulkkanen received the Ph.D. degree in aquatic sciences from the University of Jyväskylä, Jyväskylä, Finland, in 2013.

She is an Environmental Data Modeler with Vapo Oy Clean Waters, Jyväskylä, a startup business that provides expert, design, and construction services for the treatment of natural waters. 